\title{
Cassie-Wenzel transition of a binary liquid mixture on a nanosculptured surface
}

\author{
Swarn Lata Singh, Lothar Schimmele, and S. Dietrich \\ Max-Planck-Institut für Intelligente Systeme, D-70569 Stuttgart, Heisenbergstr. 3, Germany \\ Institut für Theoretische Physik, Universität Stuttgart, Pfaffenwaldring 57, D-70569 Stuttgart, Germany
}

(Received 22 January 2020; accepted 13 March 2020; published 15 May 2020)

\begin{abstract}
The Cassie-Wenzel transition of a symmetric binary liquid mixture in contact with a nano-corrugated wall is studied. The corrugation consists of a periodic array of nanopits with square cross sections. The substrate potential is the sum over Lennard-Jones interactions, describing the pairwise interaction between the wall particles $\mathrm{C}$ and the fluid particles. The liquid is composed of two species of particles, $\mathrm{A}$ and $\mathrm{B}$, which have the same size and equal A-A and B-B interactions. The liquid particles interact between each other also via A-B Lennard-Jones potentials. We have employed classical density functional theory to determine the equilibrium structure of binary liquid mixtures in contact with the nano-corrugated surface. Liquid intrusion into the pits is studied as a function of various system parameters such as the composition of the liquid, the strengths of various interparticle interactions, and the geometric parameters of the pits. The binary liquid mixture is taken to be at its mixed-liquid-vapor coexistence. For various sets of parameters the results obtained for the Cassie-Wenzel transition, as well as for the metastability of the two corresponding thermodynamic states, are compared with macroscopic predictions in order to check the range of validity of the macroscopic theories for systems exposed to nanoscopic confinements. Distinct from the macroscopic theory, it is found that the Cassie-Wenzel transition cannot be predicted based on the knowledge of a single parameter, such as the contact angle within the macroscopic theory.
\end{abstract}

DOI: 10.1103/PhysRevE.101.052115

\section{INTRODUCTION}

Wetting of solid surfaces by liquids is ubiquitous in nature [1-3] as well as in various technological applications [4-9]. It is well established that topography, both geometrical and chemical, plays an important role in controlling the wettability of a surface [10,11]. With the advent of advanced fabrication techniques, it is possible to tailor the topography of a surface down to the nanoscale $[12,13]$. One observes that changing the topography, even only on the nanoscale, results in large changes of macroscopic observables, such as the contact angle of a sessile drop. This has encouraged the fabrication of patterned surfaces in order to control wetting. Typical configurations, which liquids exhibit on textured surfaces, are either the Cassie-Baxter (CB) [14] or the Wenzel (W) state [15]. In the CB state (for short also named the Cassie state) liquid remains suspended above the substrate surface, whereas in the $\mathrm{W}$ state the liquid intrudes the surface cavities, i.e., pits.

The requirement of surface wettability, however, varies from one application to the other. Several manufacturing, biological, and agrochemical applications require the liquid to spread rapidly on the surface [16-19], whereas poor wettability is required in designing liquid-repellent surfaces

Published by the American Physical Society under the terms of the Creative Commons Attribution 4.0 International license. Further distribution of this work must maintain attribution to the author(s) and the published article's title, journal citation, and DOI. Open access publication funded by the Max Planck Society.
[20-22]. Modifying the surface properties is the most exploited method to achieve the desired wettability for a given system. In addition to changing the surface texture, adding a surfactant to the liquid in order to modify its wetting behavior provides additional flexibility. Surfactants are widely used to facilitate wetting of surfaces in many industrial applications such as agrochemical, textile, chemical, and pharmaceutical industries [23-26]. In certain circumstances, it is not easy to change the surface properties, so blending the liquid turns out to be a more feasible option to control the wettability. Enhancing pesticide utilization on plant leaves is such an example, in which surfactants are added to the pesticides in order to enhance their spreading on leaves [16,19,27-29].

Although wetting of solid surfaces by a liquid mixture is encountered frequently in many industrial applications and biological systems, most of the actual studies concerning wetting focus on one-component liquids. There have been very few experimental studies regarding the wetting of a solid surface by liquid mixtures [16,23,30,31] as well as only a very few theoretical investigations [32-38] thereof. A microscopic understanding of the Cassie-Wenzel transition for liquid mixtures is crucial for many application purposes. Here we present a study of the Cassie-Wenzel transition of a binary liquid mixture at a corrugated surface. The binary liquid mixture is composed of two types of particles labeled as $\mathrm{A}$ and $\mathrm{B}$ particles. For simplicity the studies here are limited to symmetric liquids, i.e., the radii of the $\mathrm{A}$ and $\mathrm{B}$ particles are the same, as well as the strengths of the A-A and B-B interactions, whereas the A-B interaction is varied. The wall is composed of $\mathrm{C}$ particles and modeled as a periodically repeated array of nanopits with square cross sections of width 
$w$. The depth of the pit is denoted as $D$, and its side walls are vertical. Without loss of generality, we consider the case that the attractive B-C interaction is stronger than the A-C interaction. Here we use classical density functional theory (DFT) as a tool to gain a microscopic understanding of the wetting transition. In order to reduce the number of independent thermodynamic variables, the following discussions are limited to thermodynamic conditions for which the liquid and vapor phases coexist in the bulk.

Young's contact angle $\theta_{Y}$ characterizes macroscopically the wetting behavior of a liquid on a planar and homogeneous solid surface:

$$
\cos \theta_{Y}=\frac{\sigma_{s v}-\sigma_{s l}}{\sigma_{l v}},
$$

where $\sigma_{s v}, \sigma_{s l}$, and $\sigma_{l v}$ are the interfacial tensions of the solidvapor, solid-liquid, and liquid-vapor interface, respectively. According to macroscopic theory, a liquid, which is at bulk liquid-vapor coexistence and is brought in contact with a structured wall, as described above consisting of square pits with vertical sides, remains in the Cassie state if $\theta_{Y}>90^{\circ}$, whereas for $\theta_{Y}<90^{\circ}$ the liquid intrudes the pits. Within this description and for the geometry considered, the CassieWenzel transition occurs at the threshold contact angle $\theta_{i}=$ $90^{\circ}$ for intrusion, irrespective of which of the various system parameters has been tuned in order to obtain a contact angle of $90^{\circ}$. The condition for intrusion at liquid-vapor coexistence can be regarded as a special case of the Kelvin-Laplace law describing intrusion also at pressures off the coexistence pressure. For a one-component fluid, deviations from this law for nanometer-sized grooves were studied by using classical DFT [39]. For the binary fluid studied here, we limit our computations to liquid-vapor coexistence at which the macroscopic prediction is particularly simple and transparent. Already under these special conditions the intrusion scenario for binary liquids is expected to be much richer than for onecomponent liquids. For a one-component liquid one might lower Young's contact angle $\theta_{Y}$ by increasing the wall-fluid interaction strength until intrusion is observed at $\theta_{i}$, which could deviate from the macroscopic prediction. For a binary liquid one has various options for choosing the protocol used in order to tune $\theta_{Y}$ until the liquid intrudes the pits. One might expect that there will be not just one value $\theta_{i}$ for a given pit geometry but that $\theta_{i}$ depends on those fluid properties and fluid-wall interaction parameters which are not used in the tuning protocol. Of course these extra dependences can be there only if the macroscopic prediction fails.

Apart from the Cassie to Wenzel transition (or intrusion transition), information about the metastability of the Cassie and Wenzel states is of high interest as well. The metastability of a liquid on a given surface could be exploited for drug delivery and for designing superhydrophobic or omniphobic surfaces $[10,40]$. If, for given thermodynamic conditions, the Cassie state is not stable on the surface, but remains metastable for a desired duration, de facto superhydrophobicity is achieved. This would diminish the challenges to design superhydrophobic surfaces. The macroscopic equation for the coexistence of the Cassie and the Wenzel state on a textured surface yields, at bulk liquid-vapor coexistence, a coexistence contact angle $\theta_{Y}^{c o}$ at which both states have the same grand canonical potential such that the Wenzel state is stable for $\theta_{Y}<\theta_{Y}^{c o}$ and the Cassie state is stable for $\theta_{Y}>\theta_{Y}^{c o}$. This coexistence contact angle depends only on the geometrical parameters of the pits, and not on details of the liquid properties. Within a macroscopic description, the relative stability of the Cassie and the Wenzel state is controlled entirely by a single macroscopic parameter, i.e., the contact angle $\theta_{Y}$. Although the same $\theta_{Y}$ can be achieved with a multitude of combinations of system parameters, on a macroscopic level all these details are irrelevant concerning the issue of the relative stability of the Cassie and the Wenzel state. For a surface endowed with pits of square cross section of width $w$ and depth $D$ as well as with vertical side walls, at liquid-vapor coexistence $\theta_{Y}^{c o}$ can be derived from macroscopic theory as outlined in Sec. IV [see Eq. (17); a similar expression for grooves was given in Ref. [8]]:

$$
\cos \theta_{Y}^{c o}=-\frac{w}{4 D+w} .
$$

For deep pits, i.e., $D / w \rightarrow \infty$, one has $\theta_{Y}^{c o}=90^{\circ}$. However, for shallow pits $\theta_{Y}^{c o}$ may attain values considerably larger than $90^{\circ}$. For contact angles $\theta_{Y}<\theta_{Y}^{c o}$, the Wenzel state is stable. However, if $\theta_{Y}^{c o}>90^{\circ}$, macroscopic theory tells that within the range $90^{\circ} \leqslant \theta_{Y}<\theta_{Y}^{c o}$ the Cassie state is still metastable, because the liquid can intrude the pits only if $\theta_{Y}<$ $90^{\circ}$. For $\theta_{Y}<90^{\circ}$, the Cassie state is unstable. For $\theta_{Y}>\theta_{Y}^{c o}$ the Cassie state is stable and the Wenzel state is metastable up to a sufficiently large contact angle (depending on the shape of the pits) above which it is unstable. For a pit with rectangular corners and at bulk fluid-vapor coexistence, the angle above which the Wenzel state is unstable is given by $\cos \theta_{u}=-\frac{1}{\sqrt{3}}$ (i.e., $\theta_{Y} \approx 125^{\circ}$ ). The value of this angle above which the corner dries follows from simple geometric considerations, which amount to constructing a planar fluid-vapor interface meeting the three walls forming the corner at the bottom at the angle $\theta_{Y}$ (independent of whether the fluid is multicomponent or not).

The aim of the present study is to test to which extent the two aforementioned macroscopic predictions, concerning the two specific contact angles $\theta_{i}$ and $\theta_{Y}^{c o}$, are valid for liquids on a nano-corrugated surface. The focus is on binary fluids rather than one-component fluids, first, because of the practical relevance of multicomponent fluids, and, second, because investigating binary fluids provides the opportunity to produce very different fluid-wall systems, each of them being tunable throughout the same range of contact angles. This amounts to a strong test of the general macroscopic prediction $\theta_{i}=90^{\circ}$ at bulk liquid-vapor coexistence. In general, for fluids in nanoconfinement a macroscopic description becomes unreliable. Therefore it is expected that the phenomena discussed above are no longer controlled by a single macroscopic parameter, such as the contact angle. Instead, one expects that more details like the fluid composition, the fluid-fluid, and the fluid-wall interactions eventually become relevant. Therefore, we have studied the Cassie-Wenzel transition of a binary liquid mixture on a nanotextured wall using density functional theory (DFT). We have analyzed extensively the intrusion as a function of the properties of the liquid (e.g., the composition of the liquid and the relative strengths of the A-A and A-B interactions), in order to assess the effect of these properties 
on the Cassie-Wenzel transition and on the relative stabilities of the two states. The other control parameters considered in this study are the strengths of the fluid-solid interactions (A-C and B-C) of the two fluid species and the dimensions of the pit. We have varied the dimensions of the pit in order to study the effect of strong confinement on the wetting behavior. For each set of parameters, we have calculated the number density profiles for both types of fluid particles for the stable and, if appropriate, the metastable configurations. Based on them we have monitored the intrusion at bulk liquid-vapor coexistence. In order to be able to compare the microscopic results with the macroscopic predictions, we have calculated the interfacial tensions at coexistence for each model studied via DFT as well as the corresponding macroscopic Young contact angle based on Young's law [Eq. (1)]. The threshold contact angle $\theta_{i}$, below which the intrusion is observed within DFT, is compared with the macroscopic predictions. In addition, in order to determine the relative stability of the Cassie and the Wenzel states we have calculated the grand canonical potential $\Omega$ for each number density configuration generated by DFT. The contact angle corresponding to the coexistence of the Cassie and Wenzel states is compared with $\theta_{Y}^{c o}$ given by Eq. (2).

The paper is organized as follows. In Sec. II we provide a detailed description of the system studied here. It is characterized completely by the texture of the surface as well as by the fluid-fluid and fluid-surface interactions. In Sec. III we give a brief introduction to the technique used for the present investigation. In Sec. IV we discuss our results and conclude in Sec. V.

\section{FLUID-FLUID INTERACTION, WALL TOPOGRAPHY, AND SUBSTRATE POTENTIAL}

We have studied binary liquid mixtures in contact with a nanostructured wall at the bottom and the bulk of the liquid at the top. The wall is endowed with a periodic array of square nanopits, with edge length $w$ and depth $D$ (see Fig. 1). The periodicity length does not play a role in what follows, and therefore it is not shown in Fig. 1. The liquid is composed of $\mathrm{A}$ and $\mathrm{B}$ particles. These particles are considered to interact via Lennard-Jones (LJ) pair potentials. In the spirit of DFT as used here and discussed in Sec. III, each pair potential is replaced by the sum of a hard sphere interaction

$$
U_{i j}^{h s}(r)= \begin{cases}\infty, & r \leqslant \sigma_{i j}=R_{i}+R_{j} \\ 0, & r>\sigma_{i j}\end{cases}
$$

and of a soft attractive part [41]

$$
U_{i j}^{\text {att }}(r)=-\epsilon_{i j} \Theta\left(2^{1 / 6} \sigma_{i j}-r\right)+\Phi_{i j}^{L J}(r) \Theta\left(r-2^{1 / 6} \sigma_{i j}\right)
$$

with the Heaviside function $\Theta$ and

$$
\Phi_{i j}^{L J}=4 \epsilon_{i j}\left[\left(\frac{\sigma_{i j}}{r}\right)^{12}-\left(\frac{\sigma_{i j}}{r}\right)^{6}\right] .
$$

Here $i$ and $j$ represent the two species A and $\mathrm{B}$ of the binary liquid mixture, $R_{i}$ is the radius of species $i$ of the fluid particles, $-\epsilon_{i j}$ is the potential depth for the $i j$-pair potential at $r=2^{1 / 6} \sigma_{i j}, r$ is the center-to-center interparticle separation, and $\sigma_{i j}$ is the distance of contact between the centers of two interacting liquid particles. For simplicity, the binary

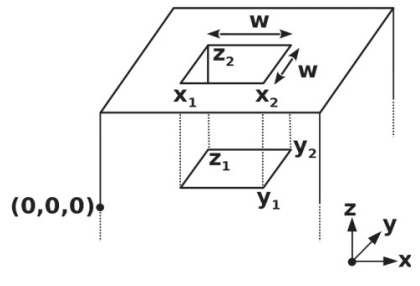

(a)

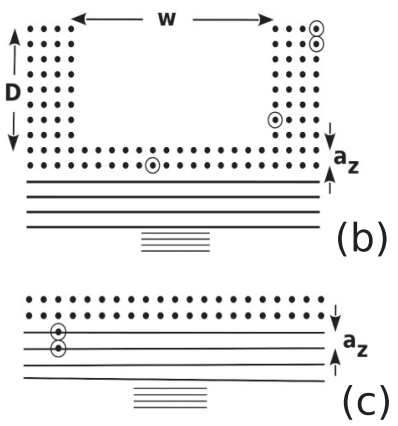

(c)
FIG. 1. (a) Schematic representation of the structured wall studied here. The structure is modeled by square pits $w \times w \times D$ of width $w$ and depth $D$. The pits are carved out from a planar wall. In (a) and (b) a single pit is shown. If the pit and a neighboring part of the wall are considered as a unit cell, which is repeated periodically in $x$ and $y$ direction, one obtains a two-dimensional lattice of the pits. The solid substrate is modeled as a simple cubic lattice occupied densely by particles of type $\mathrm{C}$. The lattice spacing is $a_{z}$, which equals twice the radius $R_{C}$ of the $\mathrm{C}$ particles. The circles indicate the size of the $\mathrm{C}$ particles, which are densely packed. In (b) the cross section of the pit is shown. For technical convenience the horizontal lattice planes below the two forming the bottom of the pit are taken to be the lateral average in the $x$ and $y$ directions of the discrete lattice structure, maintaining the discrete vertical structure. These homogeneous planes are indicated by horizontal lines; they form a half space. The liquid in the pit is sufficiently far from these homogeneous lattice planes so that their missing lateral corrugation is quantitatively negligible for the substrate potential. On the other hand, keeping the full lattice structure at the top, at the sidewalls, and at the bottom of the pit gives rise to the same local contact angle. In (c) the reference configuration of the planar substrate is shown, for which $\sigma_{s v}$ and $\sigma_{s l}$ [see Eq. (1)] are determined. The lengths $w$ and $D$ are measured between the respective loci of the nuclei of the $\mathrm{C}$ particles. These definitions imply that they encompass the corresponding depletion zone for the density profiles of the fluid particles.

liquid mixture is considered to be symmetric with $R_{A}=R_{B}$ and $\epsilon_{A A}=\epsilon_{B B}$, where $\epsilon_{i j}$ is the parameter of the interaction strength of the $i j$-pair potential. The fluid-fluid interaction is rendered effectively short-ranged by introducing a cutoff at $r=R_{c}$. In the following we adopt $R_{c}=5 R_{A}=5 R_{B}$, which is implemented by a cutoff function. The interaction parameters $\epsilon_{i j}$ for the cutoff potential are rescaled such that the integrated interaction is equal to the original one resulting from the potential in Eq. (5) with $R_{c} \rightarrow \infty$. For further details see Ref. [42].

The model for the corrugated wall is introduced and described in Fig. 1. According to Fig. 1, the very bottom of the wall consists of laterally homogenous layers (see the horizontal lines in Fig. 1) which form a half-space (see below). These layers exhibit an interlayer spacing $a_{z}=2 R_{c}$. The layers are of a macroscopic extent in the lateral $(x, y)$ directions. This half-space attracts the fluid particles via van der Waals forces A-C and B-C:

$$
U_{i}^{\text {half-space }}(r)=-4 \epsilon_{i} \sum_{l}\left[\frac{\sigma_{i}{ }^{2}}{\left|\mathbf{r}-\mathbf{r}_{l}\right|^{2}}\right]^{3},
$$


where $i=A, B$ is the fluid particle at the position $\mathbf{r}, l$ represents a half-space particle at position $\mathbf{r}_{l}$, and $\sigma_{i}$ is a (microscopic) length which can be chosen to provide the units of the number densities. In combination with the energy parameter $\epsilon_{i}$, it describes the strength of the interaction between a fluid particle of type $i$ and a wall particle. Since the layers forming the half-space are homogeneous in the lateral directions, the summation in Eq. (6) in the $x$ and $y$ directions reduces to an integration for each layer, and the contributions from the layers are summed up. For further details, we refer to Ref. [42].

The solid particles $\mathrm{C}$ occupying the remaining fully discrete, simple cubic lattice sites on top of the half-space interact with the liquid particles via a Lennard-Jones potential:

$$
U_{i}^{\text {structure }}(r)=-4 \epsilon_{i} \sum_{l}\left[\left(\frac{\sigma_{i}}{\left|\mathbf{r}-\mathbf{r}_{l}\right|}\right)^{12}-\left(\frac{\sigma_{i}}{\left|\mathbf{r}-\mathbf{r}_{l}\right|}\right)^{6}\right] .
$$

The parameters $\epsilon_{i}$ of the interaction strengths for the solidfluid pair interaction are the same as the parameters of the interaction strengths for the half-space-liquid interaction. The same holds for $\sigma_{i}$, where $\sigma_{i}=R_{i}+R_{C}, R_{i}=R_{A}=R_{B}$ is the radius of the liquid particles, and $R_{C}$ is the radius of the solid particles. In Eq. (7) the sum over $l$ amounts to the sum over all remaining discrete lattice sites occupied by $\mathrm{C}$ particles.

The total fluid-solid (fs) interaction is the sum of both contributions:

$$
U_{i}^{f s}(r)=U_{i}^{\text {half-space }}(r)+U_{i}^{\text {structure }}(r),
$$

where $i=A, B$ represents the two species of the binary liquid mixture.

\section{DENSITY FUNCTIONAL THEORY}

The grand canonical potential $\Omega$ of a classical system of an $N$-component mixture follows from a variational functional

$$
\Omega\left[\left\{\rho_{i}\right\}\right]=F\left[\left\{\rho_{i}\right\}\right]+\sum_{i=1}^{N} \int d^{3} r \rho_{i}(\mathbf{r})\left[V_{i, \text { ext }}(\mathbf{r})-\mu_{i}\right]
$$

of the one-particle number densities $\rho_{i}(\mathbf{r}), i=1, \ldots, N . F$ is the free energy functional, $V_{i, \text { ext }}(\mathbf{r})$ is the external potential, and $\mu_{i}$ is the chemical potential of species $i=A, B$, respectively. The equilibrium number densities $\rho_{i, 0}(\mathbf{r})$ minimize $\Omega$ :

$$
\left.\frac{\delta \Omega\left[\rho_{i}\right]}{\delta \rho_{i}(\mathbf{r})}\right|_{\rho_{i}(\mathbf{r})=\rho_{i, 0}(\mathbf{r})}=0 .
$$

$\Omega\left[\left\{\rho_{i, 0}\right\}\right]$ is the equilibrium grand canonical potential of the system $[43,44]$. The free energy functional consist of two parts:

$$
F\left[\left\{\rho_{i}\right\}\right]=F_{i d}\left[\left\{\rho_{i}\right\}\right]+F_{e x}\left[\left\{\rho_{i}\right\}\right],
$$

where $F_{i d}$ is the ideal gas part

$$
F_{i d}\left[\rho_{i}\right]=k_{B} T \sum_{i=1}^{N} \int d^{3} r \rho_{i}(\mathbf{r})\left[\ln \left(\rho_{i}(\mathbf{r}) \Lambda_{i}\right)-1\right],
$$

$\Lambda_{i}=\left(\frac{h^{2}}{2 \pi m_{i} k_{B} T}\right)^{3 / 2}$ is the cube of the thermal wavelength associated with a particle of species $i$ and mass $m_{i}, h$ is Planck's constant, and $k_{B}$ is the Boltzmann constant.

The excess part $F_{e x}$ arises due to the interparticle interactions. We approximate the excess part as the sum of two distinct contributions: one arising due to the hard core repulsion $\left(F_{h s}\right)$, and the other due the attractive part of the interaction $\left(F_{\text {att }}\right)$ :

$$
F_{e x}=F_{h s}+F_{\text {att }} .
$$

$F_{h s}$ is treated within the framework of fundamental measure theory (FMT), as described in the next section. $F_{\text {att }}$ is approximated within a simple random phase approximation.

\section{Fundamental measure theory}

The fundamental measure excess free-energy functional for a mixture of hard spheres, as proposed by Rosenfeld, is given by [45-47]

$$
\beta F_{h s}\left[\rho_{i}\right]=\int d^{3} r \phi\left(\left[n_{\alpha}(\mathbf{r})\right]\right),
$$

where the excess free energy density $\phi$ is a function of scalar, $n_{\alpha}(\mathbf{r})$, and vectorial, $\mathbf{n}_{\alpha}(\mathbf{r})$, weighted densities defined as

$$
n_{\alpha}(\mathbf{r})=\sum_{i=1}^{N} \int d^{3} r^{\prime} \rho_{i}\left(\mathbf{r}^{\prime}\right) \omega_{i, \alpha}\left(\mathbf{r}-\mathbf{r}^{\prime}\right)
$$

The short-hand notation in Eq. (15) should be understood such that scalar weighted densities $n_{\alpha}(\mathbf{r})$ are computed using the scalar weight functions $\omega_{i, \alpha}$ whereas vectorial weighted densities $\mathbf{n}_{\alpha}(\mathbf{r})$ are computed by using vectorial weight functions $\boldsymbol{\omega}_{i, \alpha}$. The weight functions $\omega_{i, \alpha}, \boldsymbol{\omega}_{i, \alpha}$ characterize the geometry of the spherical particles of species $i$. These weight functions are given by $[45,48]$

$$
\begin{array}{ll}
\omega_{i, 3}(\mathbf{r})=\Theta\left(R_{i}-r\right), & \omega_{i, 2}(\mathbf{r})=\delta\left(R_{i}-r\right), \\
\boldsymbol{\omega}_{i, 2}(\mathbf{r})=\frac{\mathbf{r}}{r} \delta\left(R_{i}-r\right), & \omega_{i, 1}(\mathbf{r})=\frac{\omega_{i, 2}(\mathbf{r})}{4 \pi R_{i}}, \\
\boldsymbol{\omega}_{i, 1}(\mathbf{r})=\frac{\boldsymbol{\omega}_{i, 2}(\mathbf{r})}{4 \pi R_{i}}, & \text { and } \quad \omega_{i, 0}(\mathbf{r})=\frac{\omega_{i, 2}(\mathbf{r})}{4 \pi R_{i}^{2}},
\end{array}
$$

where $R_{i}$ is the radius of the spherical particles of species $i, \Theta$ is the Heaviside step function, and $\delta$ is the Dirac $\delta$ function.

In the present study, a modification of the original Rosenfeld functional, as proposed by Rosenfeld et al. [49] and known as the modified Rosenfeld functional (MRF), has been used. Implementing this functional avoids spurious singular behaviors in situations with sharply peaked density distributions which might emerge if the original Rosenfeld functional $[45,47]$ or refined versions thereof [50] are used. On the other hand, the high accuracy of these functionals is largely preserved for the systems studied here. The free energy density $\phi$ within the MRF framework is

$$
\begin{aligned}
\phi= & -n_{0} \ln \left(1-n_{3}\right)+\frac{n_{1} n_{2}-\mathbf{n}_{1} \cdot \mathbf{n}_{2}}{1-n_{3}} \\
& +\frac{\left(n_{2}\right)^{3}}{24 \pi\left(1-n_{3}\right)^{2}}\left[1-\xi^{2}\right]^{q}
\end{aligned}
$$


where $q \geqslant 2$ and $\xi=\frac{\mathbf{n}_{2}}{n_{2}}$ [note that $\mathbf{n}_{2} \cdot \mathbf{n}_{2} \neq\left(n_{2}\right)^{2}$ ]. We have chosen $q=3$, which reproduces the original Rosenfeld functional up to the order $\xi^{2}$. In order to approximate the contribution to the free energy due to the attractive part of the interaction, we have used the following truncation of the corresponding functional perturbation expansion:

$$
F_{\text {att }}=\frac{1}{2} \sum_{i, j=1}^{N} \int d^{3} r \int d^{3} r^{\prime} \rho_{i}(\mathbf{r}) \rho_{j}\left(\mathbf{r}^{\prime}\right) U_{i j}^{\text {att }}\left(\mathbf{r}-\mathbf{r}^{\prime}\right),
$$

with $U_{i j}^{\text {att }}$ defined via Eqs. (4) and (5). Here the minimization of $\Omega\left[\left\{\rho_{i}\right\}\right]$ has to be carried out numerically. The number density is discretized on a regular simple cubic grid, and a Piccard iteration scheme is used in order to minimize $\Omega$ and to determine the equilibrium number densities. The weighted densities are calculated in Fourier space using the convolution theorem. Further details about these techniques can be found in Refs. [42,48]. The distributions $\delta$ and $\Theta$ have been smeared out in order to achieve stable convergence for the convolution (for details see Ref. [42]).

\section{COMPUTATIONAL DETAILS AND RESULTS}

In this section we present the results of DFT calculations concerning the intrusion of a binary liquid mixture at liquidvapor coexistence in the bulk into the pits of a corrugated wall. These calculations have been carried out in a computational box, the linear dimensions of which in the $x, y$, and $z$ directions have been chosen as $26 R_{A} \times 26 R_{A} \times 40 R_{A}$ (with the radii $R_{A}$ and $R_{B}=R_{A}$ of the species $\mathrm{A}$ and $\mathrm{B}$, respectively).

The corrugated wall containing pits with a square cross section (see Sec. II) has been placed at the bottom of the computational box with the normal of the wall pointing in the $z$ direction. The radius of the wall particles has been chosen as $R_{C}=R_{A} / 3$. This choice of the size of the wall particles turns out to be a sound compromise between minimizing the effect of wall roughness on interfacial structures and the computational cost. Periodic boundary conditions have been applied in the $x$ and $y$ directions. At the upper end of the box the boundary conditions prescribed for the number densities $\rho_{A}$ and $\rho_{B}$ correspond to the liquid side of liquid-vapor coexistence of the bulk of the binary liquid mixture. Alternatively to using the number densities, we also introduce the concentrations $c_{A}=\rho_{A} /\left(\rho_{A}+\rho_{B}\right), c_{B}=\rho_{B} /\left(\rho_{A}+\rho_{B}\right)$, and the total fluid packing fraction $\eta=\eta_{A}+\eta_{B}$ with $\eta_{A}=(4 \pi / 3) R_{A}^{3} \rho_{A}$, $\eta_{B}=(4 \pi / 3) R_{B}^{3} \rho_{B} ; \eta_{A}$ and $\eta_{B}$ are the fluid volume fractions blocked by the hard cores of the $\mathrm{A}$ and $\mathrm{B}$ fluid particles, respectively.

In the following calculations at various concentrations $c_{B}$ $\left(c_{A}=1-c_{B}\right)$, the concentrations in the bulk liquid adjacent to the corrugated wall always have been controlled. The wall in the computational box contains a single pit. The periodic boundary conditions imposed in the $x$ and $y$ directions imply, however, that a wall with a periodic array of pits is considered. But in the calculations presented here, the distance between the pits in the array is sufficiently large so that de facto liquid intrusion into isolated pits is studied.

The strength of the interaction between like particles has been kept fixed in all studies presented here, such that $\epsilon_{A A} /\left(k_{B} T\right)=0.9834\left(T\right.$ is the absolute temperature and $k_{B}$ is the Boltzmann constant). In most of our studies the pits have a width of $w=7 \sigma$ and a depth of $D=4 \sigma\left(\sigma=2 R_{A}=\right.$ $2 R_{B}$ is the diameter of the fluid particles); i.e., in the case of molecular fluids they are truly nanoscopic. Studying narrow and shallow pits reduces the computational costs and also opens the opportunity to observe specific effects due to nanoconfinement.

In a first series of computations, a fluid has been studied which effectively behaves as a one-component fluid with respect to bulk properties. For this fluid model the fluidfluid interaction strengths are all equal, i.e., $\epsilon_{A A}=\epsilon_{A B}=\epsilon_{B B}$. However, the $\mathrm{A}$ and the $\mathrm{B}$ particles are taken to interact with the solid wall with different strengths. The B particles are chosen to interact more strongly with the wall than the A particles. Although such a system might not be realizable in practice, the model complements the spectrum of fluid-wall systems.

The model may also be considered to mimic a bona fide one-component fluid interacting with a wall in a more complex way than via a Lennard-Jones potential characterized by a single interaction strength. The enrichment of B particles close to a wall leads to an enhanced effective fluid-wall interaction at short distances. The additional degree of freedom allows one to study, even for a "one-component" fluid, two different protocols which can be followed in order to trigger liquid intrusion and to study the relative stabilities of the Wenzel and the Cassie states involved.

In Sec. IV A 1 studies are presented for which the interaction parameter $\epsilon_{A}$ of the A particles with the wall has been fixed to such a value that the planar wall is lyophobic for a pure A fluid, i.e., the macroscopic contact angle characterizing this fluid-wall system is $\approx 112^{\circ}$. The strength $\epsilon_{B}$ of the interaction of the $B$ particles with the wall has been fixed, too, such that $\epsilon_{B} / \epsilon_{A}=2.33$. By changing the concentration $c_{B}$ of the $\mathrm{B}$ particles in the fluid, the macroscopic contact angle $\theta_{Y}$ can be tuned to values smaller than the one for the pure A fluid, due to the stronger interaction of the B particles with the wall. Thus, at bulk liquid-vapor coexistence, intrusion of the liquid into the pits can be studied as function of $c_{B}$. Furthermore, one can test the (meta)stability of the intruded Wenzel state versus the Cassie state, in which the liquid remains above the pit and the pit is filled with its vapor. In order to test the metastability, the iterative determination of the density distribution is typically initialized with two different configurations. The first one is similar to the Cassie configuration with bulk liquid densities above the pit and bulk vapor densities inside the pit with the liquid-vapor interface placed close to the opening of the pit. The second initial configuration is one with the bulk liquid occupying the whole accessible space.

Another route to vary $\theta_{Y}$, within the special fluid model chosen in the above first series of computations, consists of changing the interaction strengths $\epsilon_{A}$ and $\epsilon_{B}$ of the fluidwall interaction. The results of these studies are presented in Sec. IV A 2. In all these studies the outcome of the microscopic DFT calculations concerning the intrusion is compared with the macroscopic prediction, which, at bulk liquid-vapor coexistence, simply states that for $\theta_{Y}<90^{\circ}$ the liquid intrudes the pit. Furthermore, the relative stability of the Wenzel and the Cassie states and their free-energy difference [provided that both states do occur as (meta)stable states] are computed 

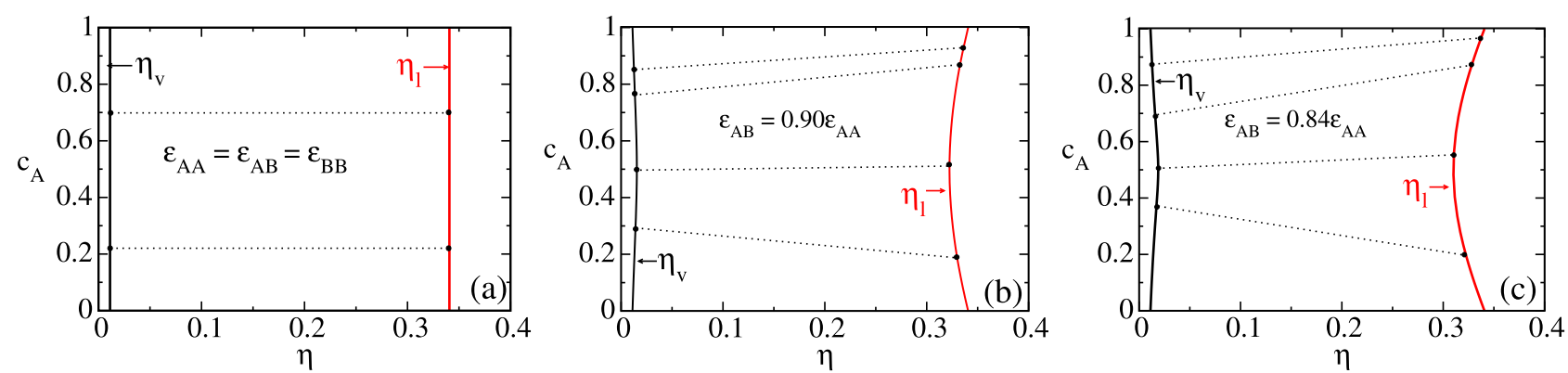

FIG. 2. Phase diagrams of the binary liquid mixture in the plane of total packing fraction $\eta$ and concentration $c_{A}$ of A particles $\left(c_{B}=1-c_{A}\right)$ for various ratios $\epsilon_{A B} / \epsilon_{A A}\left(\epsilon_{A A}=\epsilon_{B B}\right.$ in all cases). The temperature is fixed such that $\epsilon_{A A} /\left(k_{B} T\right)=0.9834$. The black and the red lines show the packing fractions at bulk coexistence in the vapor and in the liquid phase, respectively, as a function of $c_{A}$. Panel (a) corresponds to $\epsilon_{A B}=\epsilon_{A A}=\epsilon_{B B}$, (b) to $\epsilon_{A B}=0.90 \times \epsilon_{A A}$, and (c) to $\epsilon_{A B}=0.84 \times \epsilon_{A A}$. For $\epsilon_{A B} / \epsilon_{A A} \neq 1$, the concentrations $c_{A}$ and $c_{B}$ in the coexisting liquid (l) and vapor (v) phases, respectively, are different. This is demonstrated by the tilt of the dotted tielines.

using both microscopic DFT as well as the macroscopic theory. The input parameters needed for the macroscopic theory are readily computed by carrying out independent calculations of the various interfacial tensions at planar interfaces [using Young's law in Eq. (1) in order to determine $\theta_{Y}$ ] for the very same microscopic model and the identical parameter set as used in the DFT studies for the full problem of liquid intrusion.

A key issue is, if, based on the value of Young's contact angle alone, one can predict whether a liquid intrudes a pit or not, or whether additional microscopic parameters, not explicitly accounted for in a macroscopic theory, play a major role. In order to shed additional light on this question we analyze the microscopic details of the fluid structure and how these change as a result of variations of those parameters which are used to tune the contact angle $\theta_{Y}$. For instance, for a ratio $\epsilon_{B} / \epsilon_{A}$ substantially larger than one, the concentration of B particles is considerably enhanced at the walls as compared to the bulk liquid. On the other hand, for the special fluid discussed above, which is a one-component fluid with respect to bulk properties, the concentration of B particles is the same in the liquid and the vapor phase as well as across a liquidvapor interface, away from any wall. One could expect that such peculiarities, which are specific to a particular fluid-wall system, should be strongly relevant for determining the intrusion conditions, and that the values of pertinent parameters are needed in addition to the information encoded in Young's contact angle $\theta_{Y}$. This expectation is heightened by the fact that the surroundings at the opening of the pit, which should be of particular importance for the intrusion process, are very different from those implicitly assumed for the computation of $\theta_{Y}$. In narrow confinement, the role of microscopic details is expected to be even more pronounced.

We have carried out further studies along this direction by conducting another series of computations in which we consider a bona fide binary liquid mixture. In this series, the interaction strength $\epsilon_{A B}$ between unlike fluid particles is taken to be different from those between like particles $\left(\epsilon_{A A}=\epsilon_{B B}\right)$. The other parameters have the same or similar values as in the first series of computations discussed above. For various ratios $\epsilon_{A B} / \epsilon_{A A}$, the concentration $c_{B}$ has been varied, which leads to a variation of $\theta_{Y}$ (changing $\epsilon_{A B} / \epsilon_{A A}$ at fixed $c_{B}$ also leads to changes in $\theta_{Y}$ ). In the present studies, ratios $\epsilon_{A B} / \epsilon_{A A}<1$ have been considered. In this case, the concentration $c_{B}$ of the $B$ particles in the vapor phase is enhanced relative to the prescribed concentration of $\mathrm{B}$ particles in the liquid phase (see Fig. 2). Accordingly, there is a gradient in $c_{B}$ across the liquidvapor interface with the higher values of $c_{B}$ occurring at the side pointing towards the pit filled with vapor. This is distinct from the situation discussed above; the modified interplay of such a profile of $c_{B}$, with $c_{B}$ enhanced at the walls, might be relevant for liquid intrusion. For various ratios $\epsilon_{A B} / \epsilon_{A A}$, in Fig. 3 we have displayed the corresponding density and concentration profiles for both the A and the B particles, respectively, across the free liquid-vapor interface. The results of these studies, in which the wall fluid interaction has been varied, are presented in Sec. IV B.

Finally, in Sec. IVC the results of certain studies are presented, in which the effects are explored due to changing the width or the depth of the pit. These studies have been
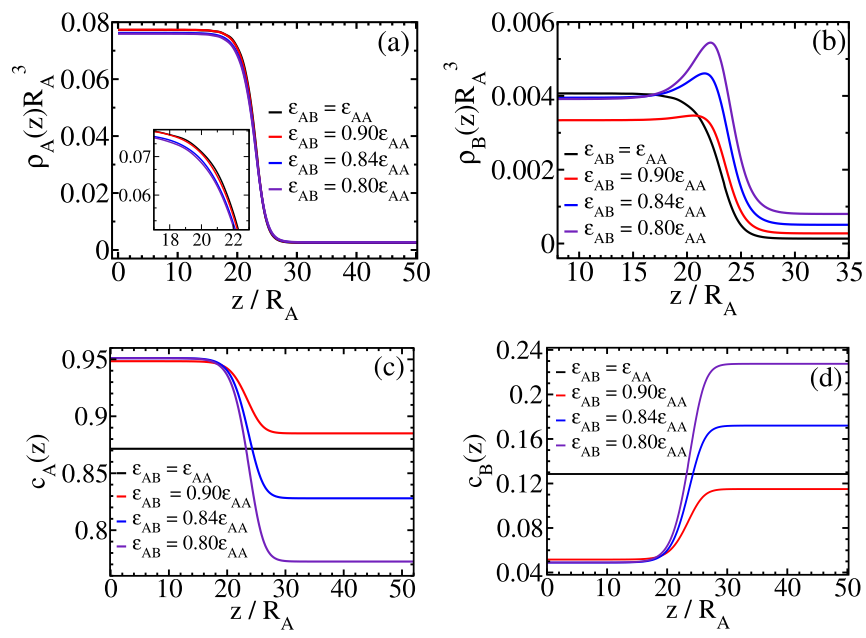

FIG. 3. Number density and concentration profiles for A and B particles across the free liquid-vapor interface for various values of $\epsilon_{A B} / \epsilon_{A A}$ and for the reduced temperature $\epsilon_{A A} /\left(k_{B} T\right)=0.9834$. The black line in panels (c) and (d) represents the concentration profiles across the liquid-vapor interface. This is spatially constant for the binary liquid mixtures with $\epsilon_{A B}=\epsilon_{A A}$, which are effectively one-component fluids so that the concentrations are the same in the vapor and liquid phases. 
carried out for the model within which the fluid in the bulk is de facto a one-component one.

\section{A. Special binary fluid: One-component in the bulk, binary with respect to the wall interactions}

In this subsection we discuss the wetting behavior of a special liquid, which is a one-component liquid in the bulk $\left(R_{A}=R_{B}, \epsilon_{A A}=\epsilon_{A B}=\epsilon_{B B}\right)$, but a binary one with respect to the interaction with the wall. The wall-liquid interactions are chosen such that the B particles are attracted more strongly by the wall than the A particles. We have varied the wall-fluid interaction strengths $\epsilon_{A}$ and $\epsilon_{B}$ as well as the ratio $\epsilon_{B} / \epsilon_{A}$. In the following, the wall-liquid interaction is represented in terms of the macroscopic Young contact angle $\theta_{Y}\left(c_{B}=0\right)$ formed by a pure A-type fluid on the corresponding planar wall. Because the properties of the pure A fluid are kept fixed in all calculations presented here, $\theta_{Y}\left(c_{B}=0\right)$ uniquely characterizes the changes of the interaction between the A particles and the wall. This choice of representation has two benefits. First, it facilitates intuition for the A particle-wall interaction strength. Second, this representation of the interaction strength is more robust with respect to numerical uncertainties resulting from discretizing the particle number densities on a grid which due to practical computational limitations cannot be refined as much that the values of all quantities have fully converged. For instance, intrusion of a pure A liquid into a pit would occur at somewhat different values of $\epsilon_{A}$ for different mesh widths of the grid; however, it occurs at the same contact angle, calculated for the respective grid width. First, in Sec. IV A 1 we discuss the intrusion as a function of composition of the liquid, keeping $\epsilon_{A}, \epsilon_{B}$, and $\epsilon_{B} / \epsilon_{A}$ fixed. In Sec. IV A 2 we study the influence of the strengths of the fluid-solid interactions, keeping the composition of the liquid fixed.

\section{Effect of composition}

In order to study how the composition of a binary liquid mixture affects its wetting behavior on a textured wall, we have fixed $\epsilon_{A B}=\epsilon_{A A}\left(=\epsilon_{B B}\right)$ and varied the composition of the liquid. We have started our analysis by considering a liquid which consists purely of A particles to which we gradually add $B$ particles until we observe intrusion. The interaction strength $\epsilon_{A}=0.9834 \times k_{B} T$ has been chosen such that the pure A liquid forms a contact angle $\theta_{Y}\left(c_{B}=0\right) \approx 112^{\circ}$. For the mixture with $c_{B} \neq 0$ a fixed ratio $\epsilon_{B} / \epsilon_{A}=2.333$ has been used. The composition in the liquid phase is controlled such that for each composition liquid-vapor coexistence is maintained. As a prerequisite the macroscopic Young contact angle $\theta_{Y}$ on a corresponding planar wall has been computed as a function of composition. Increasing the concentration of $\mathrm{B}$ particles decreases the contact angle (see Fig. 11 in Appendix A) as a result of the stronger interaction of the $\mathrm{B}$ particles with the wall. In the next step, liquid intrusion (i.e., the Cassie-Wenzel transition) into pits with square cross section is studied as function of the composition of the liquid. In order to detect the intrusion, for various compositions we have calculated within DFT the density profiles of the binary liquid mixture, always at liquid-vapor coexistence. In order to observe the Cassie-Wenzel transition, the iterative determination of the fluid density profiles is initialized with a liquid-vapor interface placed at the opening of the pits. The pits are filled with the coexisting vapor, whereas the liquid remains suspended on the top of the pits. We have determined the value of $\theta_{Y}$ below which intrusion occurs; according to the macroscopic theory this is expected to occur for $\theta_{Y}<90^{\circ}$. We also carried out calculations with initializing the system in the Wenzel state (i.e., the pits are filled with liquid) and computed the grand canonical potentials of both states, provided both are (meta)stable. These results are compared with the macroscopic predictions. At liquid-vapor coexistence, the difference between the grand canonical potentials of the Cassie-Baxter state $\left(\Omega_{C B}\right)$ and of the Wenzel state $\left(\Omega_{W}\right)$ can be computed within the macroscopic theory once the interfacial tensions are known; for a pit with square cross section of width $w$ and depth $D$ one has

$$
\Omega_{C B}-\Omega_{W}=\Delta \Omega=\sigma_{l v} w^{2}\left(1+\frac{4 D+w}{w} \cos \theta_{Y}\right) .
$$

Both states coexist, i.e., $\Omega_{C B}=\Omega_{W}$, if

$$
\cos \theta_{Y}^{c o}=-\frac{w}{4 D+w}
$$

[see Eq. (2) above.

Here we discuss results for $w=7 \times \sigma\left(\sigma=2 \times R_{A}\right)$ and $D=4 \times \sigma$. For the pure A liquid $\left(c_{B}=0\right)$, the Cassie state is the stable state. If the iteration is initialized in the Wenzel state, the latter turns out to be metastable with a higher grand canonical potential. If the liquid reaches $5 \%$ of B particles $\left(c_{B}=0.05\right)$, the previously stable Cassie state becomes metastable. The contact angle for this composition is $\theta_{Y} \approx 99^{\circ}$ (see Fig. 11 in Appendix A). For this composition the Wenzel state has a lower grand canonical potential. The metastability of the Cassie state was tested by starting the iteration scheme with a liquid-vapor interface positioned one $\sigma$ deep inside the pit. Once the iteration has converged, the liquid-vapor interface has moved up to the same position as the one obtained by using the standard Cassie state initialization in the case that the latter is stable. For $c_{B}=0.09$ the contact angle reduces to $\theta_{Y} \approx 90^{\circ}$, and the Cassie state still remains metastable. For $c_{B} \gtrsim 0.10, c_{B}$ has been ramped up in smaller steps of ca. 0.01 . The Cassie state remains metastable up to $c_{B} \approx$ 0.129 , corresponding to $\theta_{Y} \approx 83^{\circ}$. Figure 12 in Appendix A shows the density distributions for this liquid composition of $c_{B} \approx 0.129$. If the concentration of $\mathrm{B}$ particles is increased further up to $c_{B} \approx 0.138$, which corresponds to $\theta_{Y} \approx 81^{\circ}$, the Cassie state becomes unstable. Both initial configurations (Cassie and Wenzel) eventually converge into the Wenzel state (Fig. 13 in Appendix A). The number density of B particles is much higher at the walls as compared with that of the A particles. In Fig. 4 we show the difference $\Delta \Omega$ of the grand canonical potentials of the Cassie and Wenzel states, for compositions between $c_{B}=0$ and $c_{B} \approx 0.138$, as obtained from DFT. These values are compared with $\Delta \Omega$ calculated from Eq. (17) for $w=7 \times \sigma, D=4 \times \sigma$ (red line), and for an alternative convention for defining the width and the depth of the pit, i.e., $w=6 \times \sigma, D=3 \times \sigma$ (blue line). In the first convention the width is defined as the distance between the centers of the substrate particles in the topmost layers of the opposing wall surfaces, i.e., including the depletion 


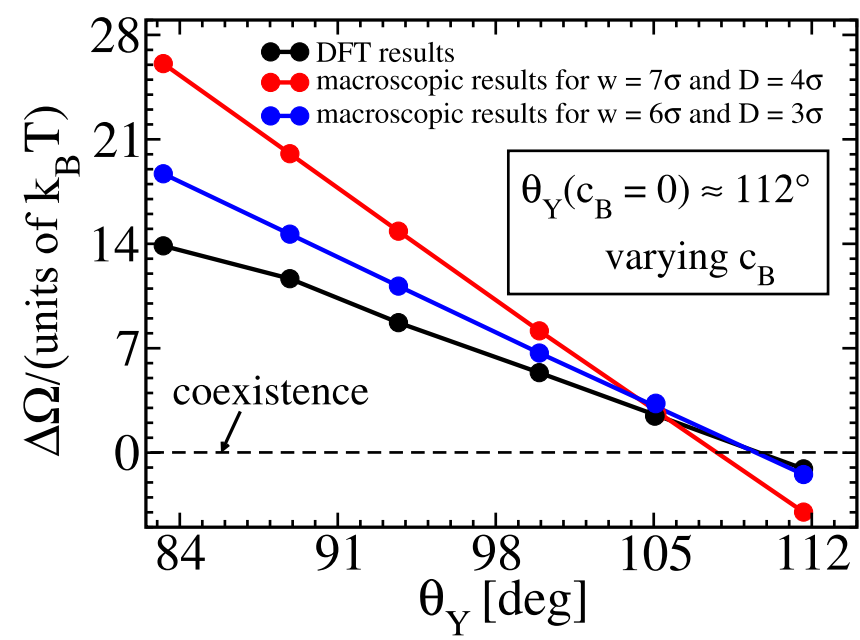

FIG. 4. Grand canonical free energy difference $\Delta \Omega=\Omega_{C B}-$ $\Omega_{W}$ for the Cassie-Baxter and Wenzel states as a function of $\theta_{Y}$. The contact angle $\theta_{Y}$ is varied by changing $c_{B}$ at fixed $\epsilon_{A A}=\epsilon_{A B}=\epsilon_{B B}$, $w=7 \times \sigma$, and $D=4 \times \sigma$. The value of $\epsilon_{A}$ has been chosen such that for $c_{B}=0$ the contact angle is $\theta_{Y}\left(c_{B}=0\right) \approx 112^{\circ}$. The black line corresponds to DFT results; the red line represents the macroscopic predictions based on Eq. (17), with the pit width $w=7 \times \sigma$ and depth $D=4 \times \sigma$. The blue line represents the macroscopic results for a pit with an effective width $w=6 \times \sigma$ and $D=3 \times \sigma$, taking into account the depletion zone and the slight intrusion of the liquid-vapor interface into the pit even in the Cassie state.

zones of the fluid densities; the depth is defined accordingly [see Fig. 1(b)]. In the second convention the width is defined as the width of the space inside the pit which is actually accessible to the fluid particles, thus the zones of strongly depleted fluid densities are not considered to be part of the pit volume. The reduced depth in the second convention accounts for the fact that the liquid-vapor interface in the Cassie state is not perfectly flat above the pit, but penetrates into the pit by $c a$. one $\sigma$, thus reducing the effective depth of the vapor-filled space. The contact angle $\theta_{Y}^{c o}$ corresponding to $\Delta \Omega=0$, as predicted by Eq. (18), is $108^{\circ}$ or $109^{\circ}$, respectively, whereas $\theta_{Y}^{c o}$ found from DFT is $109^{\circ}$. The macroscopic results for $\Delta \Omega$, based on two different conventions for the geometric parameters width and depth, differ considerably. The second convention renders a better agreement between the microscopic and the macroscopic results. However, none of the two acceptable alternative conventions for defining the geometric parameters lead to a close agreement between the macroscopic predictions for $\Delta \Omega$ and the microscopic results (see Fig. 4).

\section{Influence of the strength of the liquid-wall interaction}

In this section we discuss the influence of the strength of the liquid-wall interaction on the wetting behavior of the liquid and on the relative stability of the Cassie and Wenzel states. We consider again a particular liquid in the sense that it is a one-component fluid with respect to its bulk properties. The bulk composition of this liquid is fixed at $c_{B}=0.05$, but we vary the values of $\epsilon_{A}, \epsilon_{B}$, and $\epsilon_{B} / \epsilon_{A}$. Here we discuss the results for $w=7 \times \sigma$ and $D=4 \times \sigma$. We have considered two values of $\epsilon_{A}$, and for each $\epsilon_{A}$ we have varied $\epsilon_{B} / \epsilon_{A}$. First,

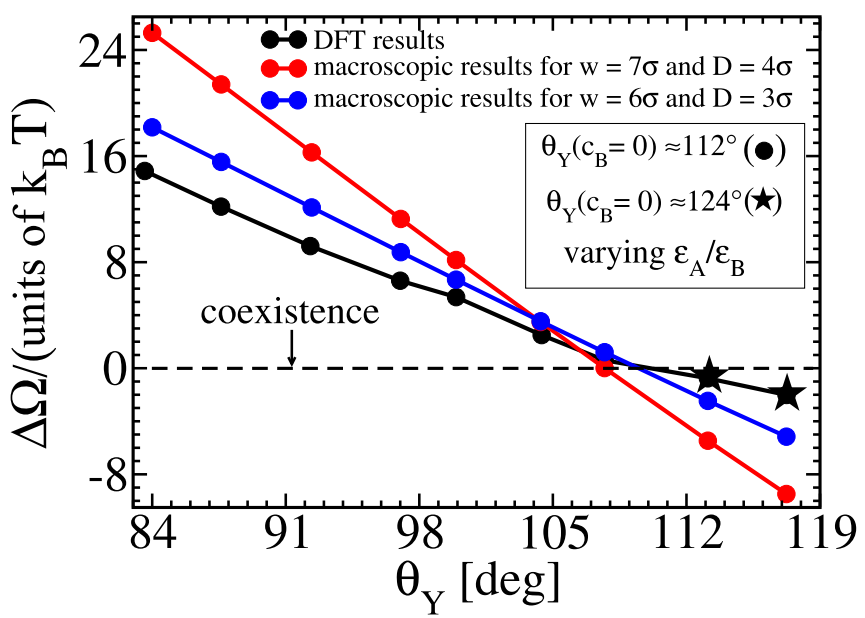

FIG. 5. Grand canonical free energy difference $\Delta \Omega=\Omega_{C B}-$ $\Omega_{W}$ for the Cassie-Baxter and Wenzel states as function of $\theta_{Y}$. The macroscopic contact angle $\theta_{Y}$ has been varied by changing $\epsilon_{A}$ and $\epsilon_{B} / \epsilon_{A}$. The black line shows DFT results, whereas the red and the blue lines correspond to the macroscopic predictions for the actual and the effective values of $w$ and $D$, respectively. We have chosen two values of $\epsilon_{A}$ resulting in $\theta_{Y} \approx 112^{\circ}$ (shown by filled circles) and $\theta_{Y} \approx 124^{\circ}$ (shown by stars) for a pure A liquid. For each of these two values of $\epsilon_{A}, \epsilon_{B} / \epsilon_{A}$ is varied in order to change $\theta_{Y}$. The other parameters are fixed at $c_{B}=0.05, \epsilon_{A A}=\epsilon_{A B}=\epsilon_{B B}, w=7 \times \sigma$, and $D=4 \times \sigma$.

we have taken the same value of $\epsilon_{A}$ as in the previous section, which leads to $\theta_{Y} \approx 112^{\circ}$ for $c_{B}=0$. We have calculated the fluid number densities and the grand canonical potential by starting the iteration scheme both from Cassie and from Wenzel configurations. For $\epsilon_{B} / \epsilon_{A}>2.833$ (i.e., $\theta_{Y}<83^{\circ}$ ), the only stable state is the Wenzel state, whereas the Cassie state is unstable. For $\epsilon_{B} / \epsilon_{A}=2.833$ (i.e., $\theta_{Y} \approx 83^{\circ}$ ), the Cassie state becomes metastable and the Wenzel state is stable with a lower grand canonical potential. We have decreased gradually the ratio $\epsilon_{B} / \epsilon_{A}$, keeping $\epsilon_{A}$ fixed, and thus increased the contact angle gradually up to $\approx 107^{\circ}$. The resulting values of $\Delta \Omega$ are represented by the filled circles in Fig. 5 . The Cassie state remains metastable up to the highest contact angle $\theta_{Y} \approx 107^{\circ}$ investigated in this series of computations.

In the next step we have changed $\epsilon_{A}$ such that $\theta_{Y}$ associated with a pure A liquid is $c a .124^{\circ}$. For this value of $\epsilon_{A}$, we present results for the two ratios $\epsilon_{B} / \epsilon_{A}=2.6$ and $\epsilon_{B} / \epsilon_{A}=2.4$, indicated by stars in Fig. 5. For $\epsilon_{B} / \epsilon_{A}=2.6$, the corresponding contact angle is $c a$. $113^{\circ}$. For this value of $\theta_{Y}$, the Cassie state becomes stable whereas the Wenzel state becomes metastable. If $\epsilon_{B} / \epsilon_{A}$ is reduced to 2.4 , the contact angle increases to $c a .117^{\circ}$ with the Wenzel state still remaining metastable. When $\epsilon_{B} / \epsilon_{A}$ is reduced further, the Wenzel state becomes unstable and the only stable state is the Cassie state.

We have compared these results of fully microscopic DFT computations (data shown in black) with the results obtained from Eq. (17). The values of $\Delta \Omega$, obtained both microscopically and macroscopically, are shown in Fig. 5. The macroscopic calculations are carried out for two sets of values for $w$ and $D$, one for the actual pit dimensions $(w=7 \times \sigma$, 
$D=4 \times \sigma$; data shown in red) and one for the effective pit dimensions ( $w=6 \times \sigma, D=3 \times \sigma$; data shown in blue). Using the effective pit dimensions improves the agreement with the DFT results, but there are still considerable discrepancies. The microscopic computations show that the Cassie and Wenzel states coexist at $\theta_{Y} \approx 110^{\circ}$. Macroscopically, the coexistence of the Cassie and the Wenzel states for the actual $(w=7 \times \sigma, D=4 \times \sigma)$ and for the effective pit dimensions $(w=6 \times \sigma, D=3 \times \sigma)$ occurs at $\theta_{Y} \approx 108^{\circ}$ and $\theta_{Y} \approx 109^{\circ}$, respectively.

\section{B. Binary liquid}

In this subsection, we study the wetting behavior as well as the metastability of the Cassie and Wenzel states for a liquid which is a bona fide binary liquid mixture also with respect to its bulk properties; but as a function of various parameters it is taken to be symmetric upon interchanging $A$ and $B\left(R_{A}=R_{B}, \epsilon_{B B}=\epsilon_{A A}\right.$, but $\left.\epsilon_{A B} \neq \epsilon_{A A}\right)$. In Sec. IV B 1 we discuss the effect of varying $\epsilon_{A B}$, keeping the liquid-wall interaction and the composition of the liquid fixed. In the subsequent Sec. IV B 2 we study the intrusion of the liquid as a function of $c_{B}$ for various values of $\epsilon_{A B}$ at fixed liquid-wall interaction. In Sec. IV B 3 we analyze the influence of the liquid-wall interaction on the wetting behavior of the binary liquid mixture.

\section{Influence of $\epsilon_{A B}$}

In order to study the influence of $\epsilon_{A B}$ on the wetting behavior, we have fixed the composition of the liquid at $c_{B} \approx$ 0.05 , the A-A and B-B interaction strengths at $\epsilon_{A A} /\left(k_{B} T\right)=$ $\epsilon_{B B} /\left(k_{B} T\right)=0.9834$, and decreased the ratio $\epsilon_{A B} / \epsilon_{A A}$ from 1.0 , in steps of 0.05 , to $\epsilon_{A B} / \epsilon_{A A}=0.90$, and then from $\epsilon_{A B} / \epsilon_{A A}=0.90$, in smaller steps of 0.02 , to $\epsilon_{A B} / \epsilon_{A A}=0.80$. For $\epsilon_{A B}=0.90 \times \epsilon_{A A}$ and $\epsilon_{A B}=0.84 \times \epsilon_{A A}$, the phase diagram of the liquid as a function of $c_{A}=1-c_{B}$, is shown in Fig. 2. For $\epsilon_{A B} / \epsilon_{A A} \neq 1.0$, the composition of the coexisting vapor phase is not the same as that of the liquid phase, i.e., the tielines are not horizontal (Fig. 2). Furthermore the composition varies across the free liquid-vapor interface as shown in Fig. 3. The fluid-wall interaction strengths $\epsilon_{A}$ and $\epsilon_{B}$ are kept the same as in Sec. IV A 1. As a prerequisite, Young's contact angle $\theta_{Y}$ on a planar wall is determined as a function of $\epsilon_{A B}$. In Fig. 14 in Appendix B, $\theta_{Y}$ is shown as a function of $\epsilon_{A B}$ for fixed composition $c_{B} \approx 0.05$. Upon lowering $\epsilon_{A B} / \epsilon_{A A}$, the contact angle decreases from $\theta_{Y} \approx 99^{\circ}$ at $\epsilon_{A B}=\epsilon_{A A}$ to $\theta_{Y} \approx 84^{\circ}$ for $\epsilon_{A B}=0.80 \times \epsilon_{A A}$.

Next, we discuss the conditions for intrusion of the liquid into the pits with $w=7 \times \sigma$ and $D=4 \times \sigma$. From the studies presented in Sec. IV A 1 , it is known that for $c_{B}=$ 0.05 and $\epsilon_{A B}=\epsilon_{A A}$ the Cassie state is metastable and the Wenzel state is stable. If $\epsilon_{A B}$ is decreased, the Cassie state remains metastable up to $\epsilon_{A B}=0.86 \times \epsilon_{A A}$ (corresponding to $\theta_{Y} \approx 91^{\circ}$ ). For $\epsilon_{A B}=0.84 \times \epsilon_{A A}$ (corresponding to $\theta_{Y} \approx$ $89^{\circ}$ ), the Cassie state turns unstable and the Wenzel state becomes the only stable state, to which the iteration procedure converges for any initial condition. In Fig. 6 we compare the grand canonical potential difference $\Delta \Omega=\Omega_{C B}-\Omega_{W}$ obtained within DFT, with $\Delta \Omega$ calculated from Eq. (17) for

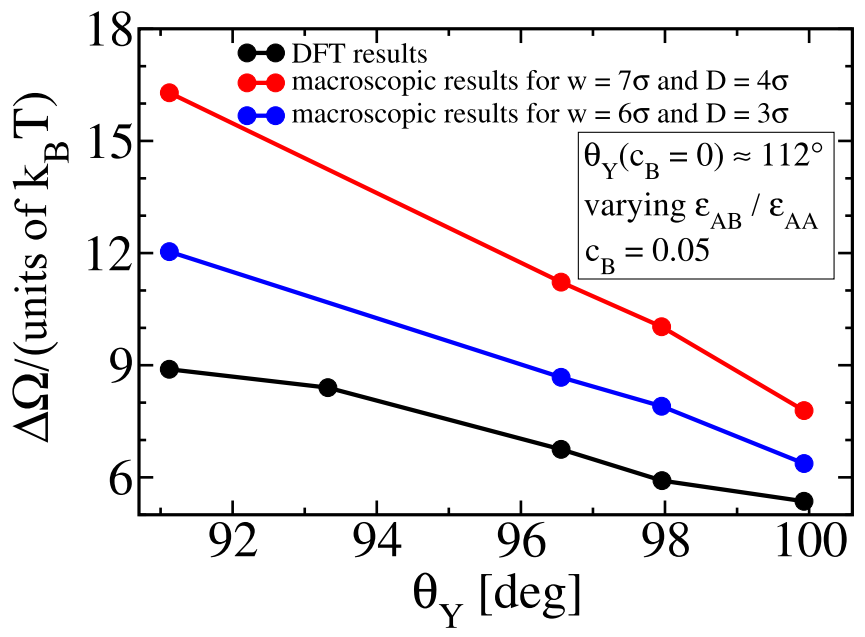

FIG. 6. Grand canonical free energy difference $\Delta \Omega=\Omega_{C B}-$ $\Omega_{W}$ for the Cassie-Baxter and Wenzel states as function of $\epsilon_{A B}$ (expressed in terms of $\theta_{Y}$; see Fig. 14), for $\epsilon_{A A}=\epsilon_{B B}$, and $c_{B}=0.05$. The black line interpolates the DFT results, whereas the red and blue lines represent $\Delta \Omega$ as obtained from the macroscopic theory for the actual parameter values $w=7 \times \sigma, D=4 \times \sigma$ and the effective ones $w=6 \times \sigma, D=3 \times \sigma$, respectively. The interaction strength $\epsilon_{A}=0.9834 \times k_{B} T$ of the A particles with the wall is chosen such that the contact angle formed by the pure A liquid on the corresponding planar wall is $\theta_{Y}\left(c_{B}=0\right) \approx 112^{\circ}$. The relative strength of the wall interactions is fixed at $\epsilon_{B} / \epsilon_{A}=2.333$.

various values of $\epsilon_{A B}$, expressed in terms of the corresponding contact angle $\theta_{Y}$.

\section{Intrusion as a function of the concentration $c_{B}$ for various interaction strengths $\epsilon_{A B}$}

In the study presented in this section, we consider various values of $\epsilon_{A B}$. For each value of $\epsilon_{A B}$ we start from conditions for which the Cassie state is metastable and reduce $\theta_{Y}$ by increasing the concentration $c_{B}$ of $\mathrm{B}$ particles until the Cassie state becomes unstable and the liquid intrudes, i.e., the pit is filled with liquid and the Wenzel state is formed. For $\epsilon_{A B}=0.90 \times \epsilon_{A A}, w=7 \times \sigma$, and $D=4 \times \sigma$ the Cassie state is metastable for $c_{B} \approx 0.05$ whereas the Wenzel state is the stable state (Sec. IV B 1 ). We have increased $c_{B}$ from ca. 0.05 onwards in steps of $\approx 0.01$. The Cassie state remains metastable up to $c_{B} \approx 0.075$, for which the Wenzel state is the stable one. The corresponding Young's contact angle is $c a .86^{\circ}$. These values correspond to the number density profiles shown in Fig. 15 in Appendix B. Upon increasing $c_{B}$ beyond 0.085 (Fig. 16 in Appendix B), thereby decreasing $\theta_{Y}$ to $84^{\circ}$, the Cassie state becomes unstable.

Upon decreasing $\epsilon_{A B}$ to $0.87 \times \epsilon_{A A}$ and keeping all other parameters fixed, the liquid intrudes the pits at a lower concentration $c_{B} \approx 0.064$ but at a higher contact angle $\theta_{Y}$ (see Fig. 7). For $\epsilon_{A B}=0.84 \times \epsilon_{A A}$, the Cassie state remains unstable for concentrations of B particles higher than $c_{B}=0.037$; at the next smaller concentration tested, $c_{B} \approx 0.025$, it becomes metastable. The corresponding contact angles are $\theta_{Y} \approx 94^{\circ}$ for $c_{B} \approx 0.037$ and $\theta_{Y} \approx 96^{\circ}$ for $c_{B} \approx 0.025$. In Fig. 7 we have introduced the threshold contact angle $\theta_{i}$ for intrusion as the mean of the largest contact angle $\theta_{Y}$ below which 


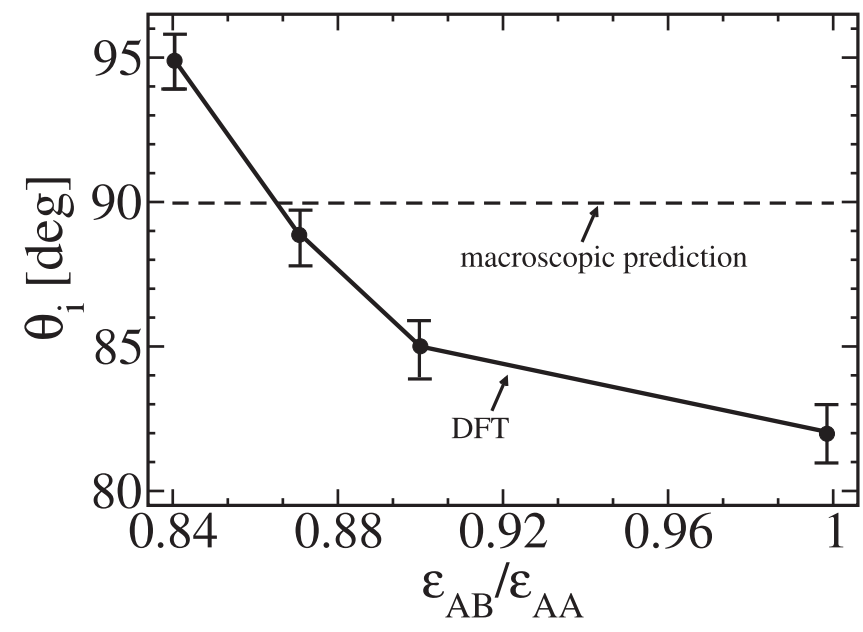

FIG. 7. Critical contact angle $\theta_{i}$, below which the Cassie state becomes unstable and liquid intrudes into the pits for four ratios $\epsilon_{A B} / \epsilon_{A A}$. For each ratio the contact angle is varied by changing the concentration $c_{B}$ such that intrusion occurs at $\theta_{Y}=\theta_{i}$. The strength $\epsilon_{A}$ is chosen such that the contact angle formed by the pure A liquid is $112^{\circ}$ and $\epsilon_{B} / \epsilon_{A}=2.333$. The pit width is $w=7 \times \sigma$ and the depth is $D=4 \times \sigma$. The critical contact angles $\theta_{i} \approx 82^{\circ}, 85^{\circ}, 89^{\circ}$, and $95^{\circ}$ correspond to $\epsilon_{A B} / \epsilon_{A A}=1.0,0.90,0.87$, and 0.84 , respectively.

intrusion is observed and the smallest angle $\theta_{Y}$ at and above which the Cassie state is found to be metastable. This gap amounts to $c a .2^{\circ}$ in the present studies (the steps in which $\theta_{Y}$ is varied) and defines the error bars indicated in Fig. 7. Obviously the threshold contact angle $\theta_{i}$ of intrusion increases upon decreasing the ratio $\epsilon_{A B} / \epsilon_{A A}$ and is not fixed at $90^{\circ}$ as predicted by the macroscopic theory.

\section{Influence of the strength of the liquid-wall interaction}

Here the studies reported in Sec. IV A 2 concerning the influence of the strength of the liquid-wall interaction on the relative stability of the Cassie and the Wenzel states are extended to ratios $\epsilon_{A B} / \epsilon_{A A} \neq 1$. For all the analyses in the present section, the pit dimensions are kept fixed at $w=$ $7 \times \sigma$ and $D=4 \times \sigma$, and the concentration of $\mathrm{B}$ particles in the bulk is always taken to be $c_{B} \approx 0.05$. Two ratios $\epsilon_{A B} / \epsilon_{A A}$ (0.9, Fig. 8) and (0.84, Fig. 9) are considered, and in each case the fluid-wall interaction strength $\epsilon_{A}$ and the ratio $\epsilon_{B} / \epsilon_{A}$ of the two fluid-wall interaction strengths have been varied. We start by discussing the case of $\epsilon_{A B} / \epsilon_{A A}=0.90$ and consider three values of $\epsilon_{A}$ and various ratios $\epsilon_{B} / \epsilon_{A}$ for each $\epsilon_{A}$.

First, we have chosen $\epsilon_{A}=0.9834 \times k_{B} T$, which was considered in the previous section, resulting in a contact angle $\theta_{Y}\left(c_{B}=0\right) \approx 112^{\circ}$ for a pure A liquid. For $\epsilon_{B} / \epsilon_{A}=2.833$, the Wenzel state is the stable state, whereas the Cassie state is unstable and thus $\Delta \Omega=\Omega_{C B}-\Omega_{W}$ is undefined. Therefore, in Fig. 8 no data point appears at the corresponding contact angle $\theta_{Y}\left(c_{B}=0.05\right) \approx 84^{\circ}$. If $\epsilon_{B} / \epsilon_{A}$ is decreased to 2.766, so that $\theta_{Y}$ increases to $\theta_{Y}\left(c_{B}=0.05\right) \approx 87^{\circ}$, the Cassie state becomes metastable whereas the Wenzel state remains the stable state $(\Delta \Omega>0)$. Upon decreasing the ratio $\epsilon_{B} / \epsilon_{A}$ further to 2.333 , the Cassie state remains metastable (as reported in the previous section), and the Wenzel state remains stable,

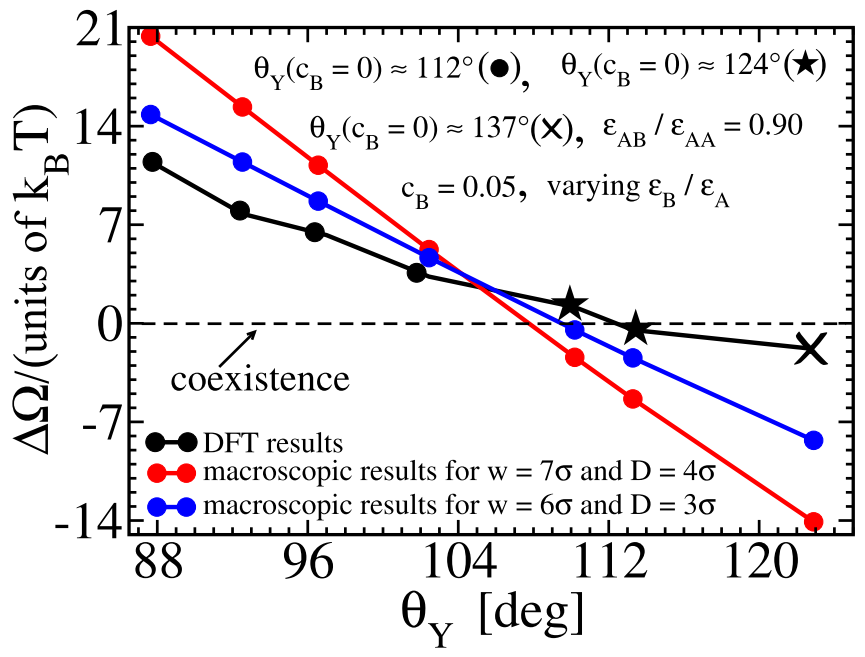

FIG. 8. Grand canonical free energy difference $\Delta \Omega=\Omega_{C B}-$ $\Omega_{W}$ for the Cassie-Baxter and Wenzel states as function of $\theta_{Y}\left(c_{B}=\right.$ 0.05 ) for $\epsilon_{A B}=0.9 \times \epsilon_{A A}$ and $c_{B}=0.05$, calculated both macroscopically (red and blue lines) and microscopically (black line). We have chosen three values of $\epsilon_{A}$, resulting in $\theta_{Y}\left(c_{B}=0\right) \approx 112^{\circ}$ (shown by filled circles), $\theta_{Y}\left(c_{B}=0\right) \approx 124^{\circ}$ (shown by stars), and $\theta_{Y}\left(c_{B}=\right.$ $0) \approx 137^{\circ}$ (shown by a cross) for a pure A liquid. For each of these values of $\epsilon_{A}, \epsilon_{B} / \epsilon_{A}$ is varied, represented in terms of a change of $\theta_{Y}$. The pit geometry is characterized by $w=7 \times \sigma$ and $D=4 \times \sigma$.

exhibiting the lower grand canonical potential $(\Delta \Omega>0)$. The resulting differences $\Delta \Omega=\Omega_{C B}-\Omega_{W}$ between the grand canonical potentials of the Cassie-Baxter and the Wenzel states (corresponding to the aforementioned first choice of $\epsilon_{A}$ and to the various values of $\epsilon_{B} / \epsilon_{A}$ considered) are shown

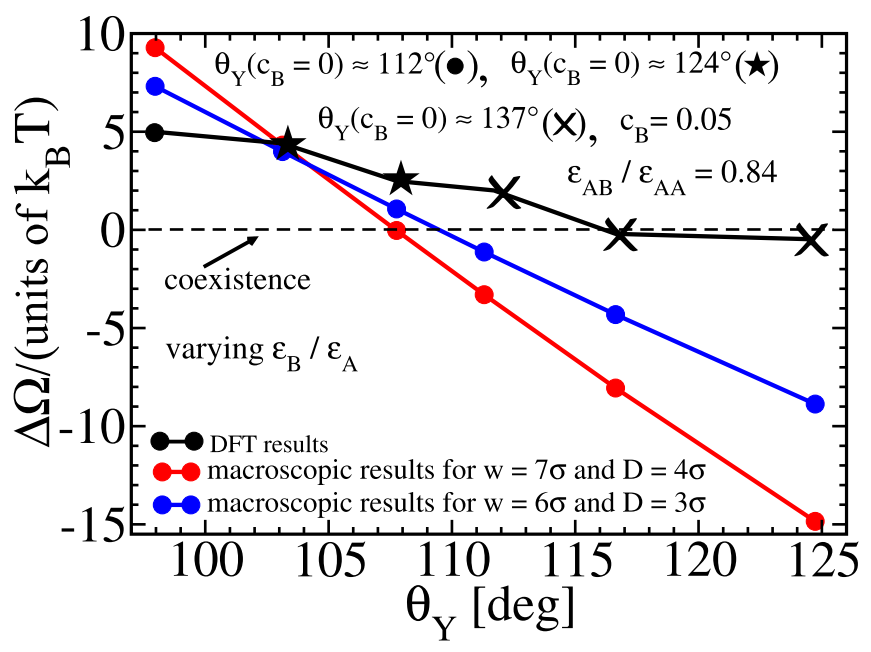

FIG. 9. Grand canonical free energy difference $\Delta \Omega=\Omega_{C B}-$ $\Omega_{W}$ for the Cassie-Baxter and Wenzel states as function of $\theta_{Y}\left(c_{B}=\right.$ $0.05)$ for $\epsilon_{A B}=0.84 \times \epsilon_{A A}$ and $c_{B} \approx 0.05$, calculated both macroscopically (red and blue lines) and microscopically (black line). The blue line corresponds to the effective (reduced) pit dimensions. We have chosen three values of $\epsilon_{A}$ corresponding to $\theta_{Y}\left(c_{B}=0\right) \approx 112^{\circ}$ (filled circles), $\theta_{Y}\left(c_{B}=0\right) \approx 124^{\circ}$ (stars), and $\theta_{Y}\left(c_{B}=0\right) \approx 137^{\circ}$ (crosses) for a pure A liquid. For each of these values of $\epsilon_{A}, \epsilon_{B} / \epsilon_{A}$ is varied, represented in terms of a change of $\theta_{Y}$. The pit geometry is characterized by $w=7 \times \sigma$ and $D=4 \times \sigma$. 
by filled circles in Fig. 8. In the second step, $\epsilon_{A}=0.8195 \times$ $k_{B} T$ is decreased such that the contact angle formed by the pure $A$ liquid on the corresponding planar wall increases to $\theta_{Y}\left(c_{B}=0\right) \approx 124^{\circ}$. The resulting values of $\Delta \Omega$ are shown by stars in Fig. 8. For $\epsilon_{B} / \epsilon_{A}=2.6$, with a corresponding contact angle $\theta_{Y}\left(c_{B}=0.05\right) \approx 110^{\circ}$, the Cassie state still remains metastable and the Wenzel state is the stable state $(\Delta \Omega>0)$. For $\epsilon_{B} / \epsilon_{A}=2.4$, so that $\theta_{Y}\left(c_{B}=0.05\right) \approx 113^{\circ}$, the Cassie and the Wenzel states coexist $(\Delta \Omega=0)$. In the previous studies for a fluid with $\epsilon_{A B}=\epsilon_{A A}$ and various fluidwall interaction strengths $\left(\epsilon_{A}, \epsilon_{B} / \epsilon_{A}\right)$, and for the same pit dimensions, the coexistence of the Cassie and the Wenzel states has been detected at $\theta_{Y}\left(c_{B}=0.05\right) \approx 110^{\circ}$ (see Fig. 5). This implies that the contact angle $\theta_{Y}^{c o}$, at which the two states coexist, has changed by $3^{\circ}$ as a result of changing certain microscopic details of the fluid-wall system, whereas the macroscopic theory [Eq. (18)] predicts that $\theta_{Y}^{c o}$ depends only on the geometric parameters $w$ and $D$ and not on any other details.

In the third step, $\epsilon_{A}=0.6556 \times k_{B} T$ has been decreased further, such that the corresponding contact angle for a pure $A$ liquid increases to $\theta_{Y}\left(c_{B}=0\right) \approx 137^{\circ}$. For the ratio $\epsilon_{B} / \epsilon_{A}=$ 3.25 , which corresponds to $\theta_{Y}\left(c_{B}=0.05\right) \approx 123^{\circ}$ for the mixture, the Wenzel state still remains metastable and the Cassie state stable $(\Delta \Omega<0)$. In Fig. 8 the resulting value of $\Delta \Omega$ is marked by a cross. If $\theta_{Y}$ is increased further, upon decreasing $\epsilon_{B} / \epsilon_{A}$, the Wenzel state becomes unstable, and the iteration process always converges to the Cassie state, irrespective of the initial conditions of the iterative determination of the equilibrium densities.

This calculation was repeated for $\epsilon_{A B}=0.84 \times \epsilon_{A A}$, (keeping $c_{B}=0.05, w=7 \times \sigma$, and $D=4 \times \sigma$ fixed). For a value of $\epsilon_{A}$ corresponding to $\theta_{Y}\left(c_{B}=0\right) \approx 112^{\circ}$ for the pure $\mathrm{A}$ liquid, for $\epsilon_{B} / \epsilon_{A}=2.333$, and for a contact angle $\theta_{Y}\left(c_{B}=\right.$ $0.05) \approx 90^{\circ}$ the Cassie state turns out to be unstable, and the only stable state is the Wenzel state, as reported in Sec. IV B 2. Keeping $\epsilon_{A}=0.9834 \times k_{B} T$ fixed, upon decreasing $\epsilon_{B} / \epsilon_{A}$ to 2, i.e., $\theta_{Y}\left(c_{B}=0.05\right) \approx 97^{\circ}$, the Cassie state becomes metastable whereas the Wenzel state remains stable; the resulting value of $\Delta \Omega$ is shown by a filled circle in Fig. 9 .

In the next step, we reduced $\epsilon_{A}=0.8195 \times k_{B} T$ such that $\theta_{Y}\left(c_{B}=0\right) \approx 124^{\circ}$ for the pure A liquid, and we considered two ratios, $\epsilon_{B} / \epsilon_{A}=2.6$ and 2.4 , with corresponding contact angles $\theta_{Y}\left(c_{B}=0.05\right) \approx 103^{\circ}$ and $\theta_{Y}\left(c_{B}=0.05\right) \approx$ $107^{\circ}$ for the mixtures, respectively. The Cassie state remains metastable, and the Wenzel state remains stable. The corresponding values of $\Delta \Omega$ are indicated in Fig. 9 by stars. The strength $\epsilon_{A}$ has been reduced further, so that the corresponding contact angle $\theta_{Y}\left(c_{B}=0\right)$, formed by the pure A liquid, increases to $\theta_{Y}\left(c_{B}=0\right) \approx 137^{\circ}$. Computations have been carried out for four ratios $\epsilon_{B} / \epsilon_{A}=3.25,3,2.5$, and 2.375 . For $\epsilon_{B} / \epsilon_{A}=3.25\left(\theta_{Y}\left(c_{B}=0.05\right) \approx 111^{\circ}\right.$ for the mixture $)$ the Cassie state is still metastable, and the Wenzel state has still a lower grand canonical potential. For $\epsilon_{B} / \epsilon_{A}=3.0\left(\theta_{Y}\left(c_{B}=\right.\right.$ $0.05) \approx 116^{\circ}$ ) both states coexist. The Wenzel state remains metastable up to $\epsilon_{B} / \epsilon_{A}=2.5\left(\theta_{Y}\left(c_{B}=0.05\right) \approx 124^{\circ}\right)$. The value of $\Delta \Omega$ for this choice of $\epsilon_{A}$ [corresponding to $\theta_{Y}\left(c_{B}=\right.$ $0.05) \approx 137^{\circ}$ ] is indicated by a cross in Fig. 9. If $\epsilon_{B} / \epsilon_{A}$ was decreased further to 2.375 , the Wenzel state became unstable.

\section{Influence of the pit dimensions}

Here the influence of the pit dimensions on the CassieWenzel transition is discussed for the fluid-wall model studied in Secs. IV A 1 and IV A 2.

\section{Varying the pit depth $D$}

In order to reveal the effect of the depth of the pit on the Cassie-Wenzel transition, we have fixed the width at $w=$ $7 \times \sigma$, whereas the depth $D$ of the pit is changed from $4 \times \sigma$ to $6 \times \sigma$. The other parameters are chosen as in Secs. IV A 1 and IV A 2, i.e., $\epsilon_{A}$ is taken such that the pure A liquid forms a contact angle of $\theta_{Y}\left(c_{B}=0\right) \approx 112^{\circ}$, with $\epsilon_{B} / \epsilon_{A}$ fixed at 2.833 and $\epsilon_{A A}=\epsilon_{A B}=\epsilon_{B B}$. For $D=6 \times \sigma$, the Cassie state remains metastable up to the value of $c_{B} \approx 0.155$, which corresponds to $\theta_{Y}\left(c_{B} \approx 0.155\right) \approx 78^{\circ}$ of the mixture and becomes unstable for $c_{B} \approx 0.165\left(\theta_{Y}\left(c_{B} \approx 0.165\right) \approx 76^{\circ}\right.$ for the mixture $)$. The threshold contact angle for intrusion would be thus $\theta_{i}=77^{\circ}$, according to the previously given definition, which has to be compared with $\theta_{i}=82^{\circ}$ obtained for the shallower pits of a depth of $4 \times \sigma$ (see Sec. IV A 1$)$.

Equation (18), which is based on the macroscopic theory, predicts that the coexistence contact angle $\theta_{Y}^{c o}$, at which the $\mathrm{CB}$ and Wenzel states in a pit of finite depth coexist and above which the $\mathrm{CB}$ state turns into the stable state, shifts from $\theta_{Y}^{c o} \approx 108^{\circ}$ for the pit depth $D=4 \times \sigma$ and a width of $w=$ $7 \times \sigma$, to $\theta_{Y}^{c o} \approx 103^{\circ}$ if the depth is increased to $D=6 \times \sigma$ while keeping the width fixed. In order to test this prediction, the calculations of Sec. IV A 2 for pits of a depth $D=4 \times \sigma$ have been repeated for deeper pits with $D=6 \times \sigma$. As in Sec. IV A 2, Young's contact angle $\theta_{Y}$ is varied by changing the fluid-wall interactions $\epsilon_{A}$ and $\epsilon_{B}$. Furthermore, the same width $w=7 \times \sigma$ of the pit, the same concentration $c_{B}$ of $B$ particles, and the same fluid-fluid interaction parameters $\epsilon_{A A}=\epsilon_{A B}=\epsilon_{B B}=0.9834 \times k_{B} T$ have been chosen as in Sec. IV A 2. A set of calculations with two distinct values of $\epsilon_{A}$ have been carried out. For the first set, $\epsilon_{A}$ has been chosen such that Young's contact angle $\theta_{Y}$ for a pure A liquid is ca. $\theta_{Y}\left(c_{B}=0\right) \approx 112^{\circ}$. The contact angle, for the given bulk concentration $c_{B}=0.05$, is tuned by changing the ratio $\epsilon_{B} / \epsilon_{A}$. The chosen ratios are $3,2.933,2.833,2.666,2.5,2.333,2.0$, and 1.666 corresponding to values of $\theta_{Y}\left(c_{B}=0.05\right)$ ranging from about $81^{\circ}$ to $107^{\circ}$. For $\epsilon_{B} / \epsilon_{A}>2.933$, the Cassie state becomes unstable, and the only stable state for the system is the Wenzel state. For $\epsilon_{B} / \epsilon_{A}=2.933\left(\theta_{Y}\left(c_{B}=0.05\right) \approx 81^{\circ}\right.$ for the mixture), the Cassie state becomes metastable, and the Wenzel state is the stable one. The value of $\epsilon_{B} / \epsilon_{A}$ has been decreased gradually, with keeping $\epsilon_{A}$ fixed, down to $\epsilon_{B} / \epsilon_{A}=$ 1.666. For $\epsilon_{B} / \epsilon_{A}=2.0\left(\theta_{Y}\left(c_{B}=0.05\right) \approx 104^{\circ}\right)$, the grand canonical potentials for the Cassie and the Wenzel states are almost equal. For $\epsilon_{B} / \epsilon_{A}=1.666\left(\theta_{Y}\left(c_{B}=0.05\right) \approx 107^{\circ}\right)$, the Wenzel state becomes metastable and the Cassie state turns into the stable state with the lower grand canonical potential. Large contact angles are modeled by reducing $\epsilon_{A}$ to a value corresponding to $\theta_{Y}\left(c_{B}=0\right) \approx 127^{\circ}$ for a pure A fluid. Calculations have been carried out for $\epsilon_{B} / \epsilon_{A}=2.8,2.6$, and 2.4 corresponding to $\theta_{Y}\left(c_{B}=0.05\right) \approx 110^{\circ}, 113^{\circ}$, and $117^{\circ}$ for the mixture. The Wenzel state remains metastable up to $\epsilon_{B} / \epsilon_{A}=$ $2.8\left(\theta_{Y}\left(c_{B}=0.05\right) \approx 113^{\circ}\right)$. If $\epsilon_{B} / \epsilon_{A}$ is decreased further, the Wenzel state becomes unstable. At $\theta_{Y}\left(c_{B}=0.05\right) \approx 117^{\circ}$, the 


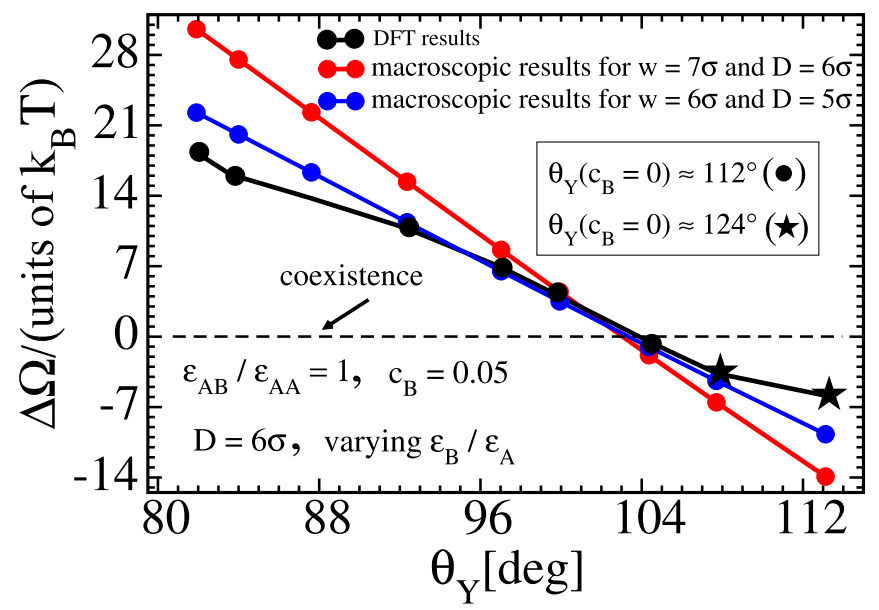

FIG. 10. $\Delta \Omega=\Omega_{C B}-\Omega_{W}$ as function of $\theta_{Y}$. The contact angle $\theta_{Y}\left(c_{B}=0.05\right)$ is varied by changing $\epsilon_{A}$ and $\epsilon_{B} / \epsilon_{A}$. The black line shows DFT results, whereas the red and the blue line correspond to the macroscopic predictions. We have chosen two values of $\epsilon_{A}$ resulting in $\theta_{Y}\left(c_{B}=0\right) \approx 112^{\circ}$ (filled circles) and $\theta_{Y}\left(c_{B}=0\right) \approx 124^{\circ}$ (stars) for a pure A liquid. For each of these two values of $\epsilon_{A}, \epsilon_{B} / \epsilon_{A}$ is changed in order to vary $\theta_{Y}\left(c_{B}=0.05\right)$. The other parameters are fixed at $c_{B}=0.05, \epsilon_{A A}=\epsilon_{A B}=\epsilon_{B B}, w=7 \times \sigma$, and $D=6 \times \sigma$.

Cassie state is the stable state. The free energy differences $\Delta \Omega$ are shown in Fig. 10 (DFT: black line). The results corresponding to the above first choice for $\epsilon_{A}$ are marked by filled circles, whereas the results corresponding to the second choice for $\epsilon_{A}$ are indicated by stars. The red and the blue lines correspond to macroscopic predictions [Eq. (17)] for the actual and the effective pit dimensions, respectively. The effective quantities $w$ and $D$ are smaller than the actual ones due to the presence of the depletion zone as well as due to the slight intrusion of the liquid-vapor interface into the pit, even in the Cassie state. Within DFT, the shift of $\theta_{Y}^{c o}$, as predicted by the macroscopic theory, is indeed observed (compare Figs. 5 and 10) and almost quantitatively agrees with the DFT results. However, away from the coexistence of the two states the DFT values of $\Delta \Omega$ deviate considerably from the macroscopic predictions.

\section{Influence of the width}

In order to study the influence of the width of the pits on the stability and metastability of the Wenzel and the Cassie states, we have fixed $c_{B}=0.05, D=4 \times \sigma, \epsilon_{A A}=\epsilon_{A B}=\epsilon_{B B}$ and decreased $w$ from $7 \times \sigma$ to $5 \times \sigma$. The value of $\epsilon_{A}$ has been chosen such that the contact angle formed by the pure A fluid is $\theta_{Y}\left(c_{B}=0\right) \approx 112^{\circ}$. Computations have been carried out for three distinct ratios $\epsilon_{B} / \epsilon_{A}=2.333,2.0$, and 1.666, corresponding to $\theta_{Y}\left(c_{B}=0.05\right) \approx 99^{\circ}, 104^{\circ}$, and $107^{\circ}$ for the mixture. For the set of parameters leading to $\theta_{Y}\left(c_{B}=0.05\right) \approx$ $99^{\circ}$, the Wenzel state is the stable state and the Cassie state is unstable. For $\theta_{Y}\left(c_{B}=0.05\right) \approx 104^{\circ}$, the Cassie and the Wenzel states coexist. For $\theta_{Y}\left(c_{B}=0.05\right) \approx 107^{\circ}$, the Wenzel state becomes unstable and the Cassie state turns into the stable state. We have repeated the same calculation for an even smaller width $w=4 \times \sigma$. For this width, the Wenzel state is the stable state with $\theta_{Y}\left(c_{B}=0.05\right) \approx 99^{\circ}$. For the next

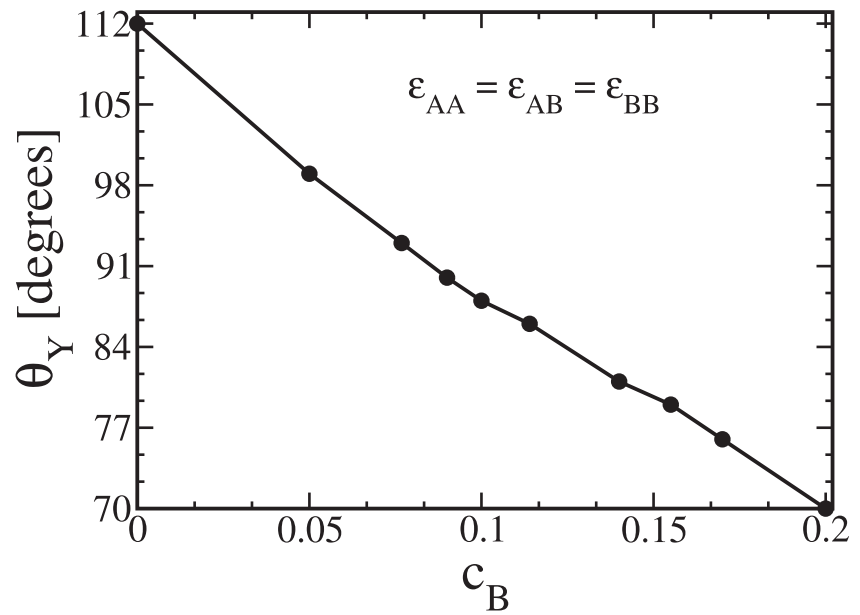

FIG. 11. Macroscopic contact angle $\theta_{Y}$ at a planar wall as function of $c_{B}$ with fixed $\epsilon_{A A}=\epsilon_{B B}=\epsilon_{A B}$. The strength $\epsilon_{A}=0.9834 \times$ $k_{B} T$ of the interaction between the wall and the A particles is chosen such that Young's contact angle attains the value $\theta_{Y} \approx 112^{\circ}$, with $\epsilon_{B} / \epsilon_{A}=2.333$ for $c_{B}=0$. The full line is a guide to the eye through the DFT data points. The absolute values of the deviations of $\theta_{Y}$ from the converged values, for a given set of interaction parameters, might be as high as $5^{\circ}$ for the grid on which the densities are discretized. The estimate is based on computations for a one component system. The relative quantity $\theta_{Y}\left(c_{B}\right)-\theta_{Y}\left(c_{B}=0\right)$, with the above fixed value of $\theta_{Y}\left(c_{B}=0\right)$, is more accurate by roughly one order of magnitude.
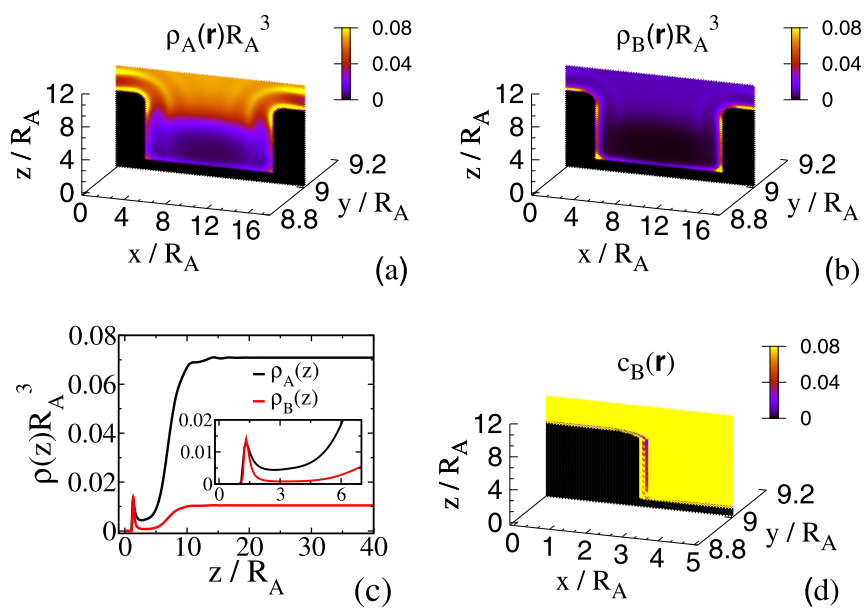

FIG. 12. Number densities $\rho_{A}(\mathbf{r})$ (a) and $\rho_{B}(\mathbf{r})$ (b) for the bulk liquid with $c_{B} \approx 0.129$ and packing fraction $\eta_{l}=0.3407$, in the $x z$ plane passing through the middle of the pit. With $\epsilon_{A A}=\epsilon_{B B}=\epsilon_{A B}$, $\epsilon_{A}$ is chosen such that $\theta_{Y}\left(c_{B}=0\right) \approx 112^{\circ}$ and with $\epsilon_{B} / \epsilon_{A}=2.333$ for $c_{B} \neq 0$. For the concentration $c_{B}$ chosen here, one has $\theta_{Y} \approx 83^{\circ}$. The depth of the pit is $D=4 \times \sigma$ and its width is $w=7 \times \sigma(\sigma=2 \times$ $R_{A}$ ). The density distributions shown here correspond to a metastable Cassie state. Panel (c) shows the densities along a line parallel to the $z$ axis and passing through the middle of the pit $\left(x=y=9 \times R_{A}\right)$. At contact with the wall the density of B particles is higher than the density of A particles. There are some density oscillations in $\rho(z)$ at the opening, which die out quickly. Panel (d) shows the concentration profile for B particles in the same plane as the one shown in panels (a) and (b), covering the left half of the pit and focusing on those parts of the pit where potentially interesting phenomena, due to variations of $c_{B}$, occur. 


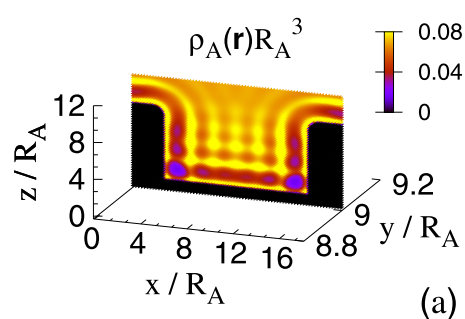

(a)

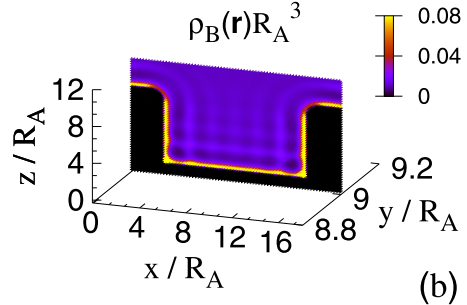

(b)

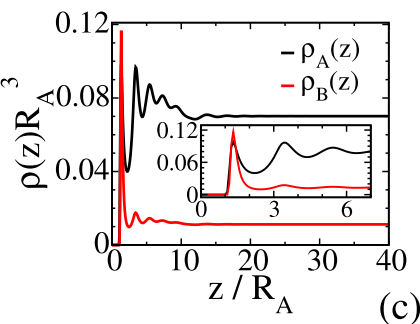

(c)

FIG. 13. Number densities $\rho_{A}(\mathbf{r})$ (a) and $\rho_{B}(\mathbf{r})$ (b) for the bulk liquid with $c_{B}=0.1378$; the other parameters are the same as in Fig. 5. The corresponding contact angle is $\theta_{Y} \approx 81^{\circ}$. The iteration scheme has been initialized in the Cassie state, which turns out to be unstable for the chosen set of parameters. Panel (c) shows the number densities along a line passing vertically through the center of the pit.

tested value $\theta_{Y}\left(c_{B}=0.05\right) \approx 104^{\circ}$, the Wenzel state becomes unstable and the Cassie state is the stable one. No metastable states have been found for $w=4 \times \sigma$.

\section{SUMMARY AND CONCLUSIONS}

We have studied the Cassie-Wenzel transition of a symmetric binary liquid mixture at a nano-corrugated surface which exhibits a periodic array of nanopits with a square cross section. For sufficiently large periodicity lengths, the case considered here, periodicity does not play a role. The liquid is composed of two types of particles labeled as A and $\mathrm{B}$. The $\mathrm{B}$ particles are taken to be attracted by the wall more strongly than the A particles. The intrusion behavior of such liquids has been studied as function of their composition, of the strength of the A-B interaction relative to the A-A and $\mathrm{B}-\mathrm{B}$ ones, of the relative strengths of the A-wall and the B-wall interactions, and of the pit dimensions. Our study has been restricted to mixed liquids at liquid-vapor coexistence. In this case the predictions of the macroscopic theory are very simple; in particular, the threshold contact angle $\theta_{i}$ for intrusion is predicted to be always $90^{\circ}$. The structural properties of the binary liquid mixtures in thermal equilibrium and, in case it applies, in metastable equilibrium have been determined by using density functional theory, which captures

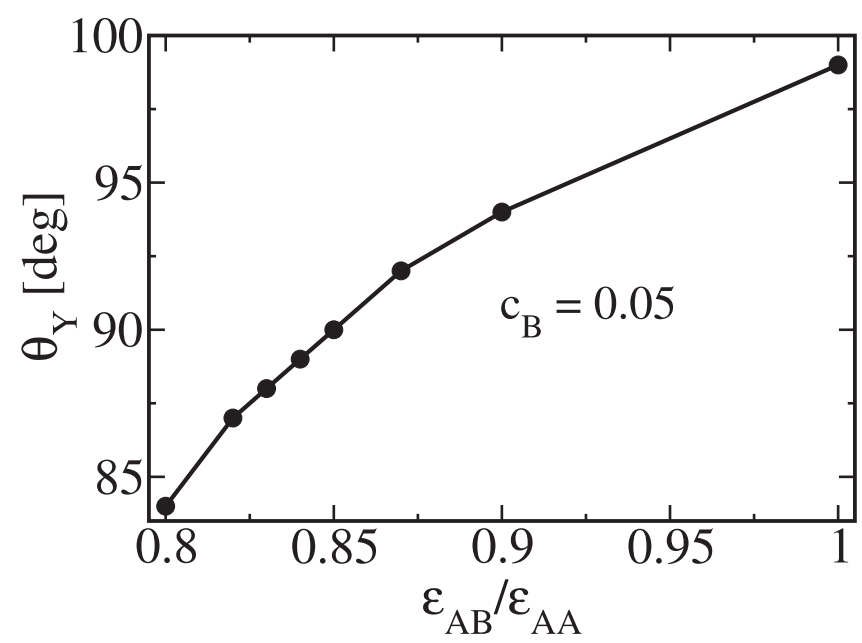

FIG. 14. Contact angle $\theta_{Y}$ as a function $\epsilon_{A B} / \epsilon_{A A}$ for $c_{B} \approx 0.05$ and $\epsilon_{A A}=\epsilon_{B B}$. The parameter $\epsilon_{A}=0.9834 \times k_{B} T$ is chosen such that $\theta_{Y} \approx 112^{\circ}$ for $c_{B}=0$, and $\epsilon_{b} / \epsilon_{A}=2.333$. The line is a guide to the eye for the DFT data points. the microscopic details of the system. The grand canonical free energy differences $\Delta \Omega$ between the stable equilibria, i.e., the Cassie or the Wenzel configuration and, as far as they occur, the competing metastable equilibria, i.e., the complementary Wenzel or Cassie configuration, respectively, have been calculated as functions of various system parameters. These results are compared with corresponding predictions from a macroscopic description in terms of surface tensions only. We have found that intrusion of the liquid into pits of given geometry, known as the Cassie-Wenzel transition, cannot be predicted reliably on the basis of a single parameter, which is the contact angle, like the macroscopic theory does. For instance, we have found that liquid intrudes a pit-of given geometry and fixed fluid-wall interactions - at a variety of contact angles if the liquid consists of symmetric binary fluids with various ratios of the A-B and A-A interaction strengths. Once, in the aforementioned case, the contact angle is reduced by increasing the concentration of $\mathrm{B}$ particles, and the liquid
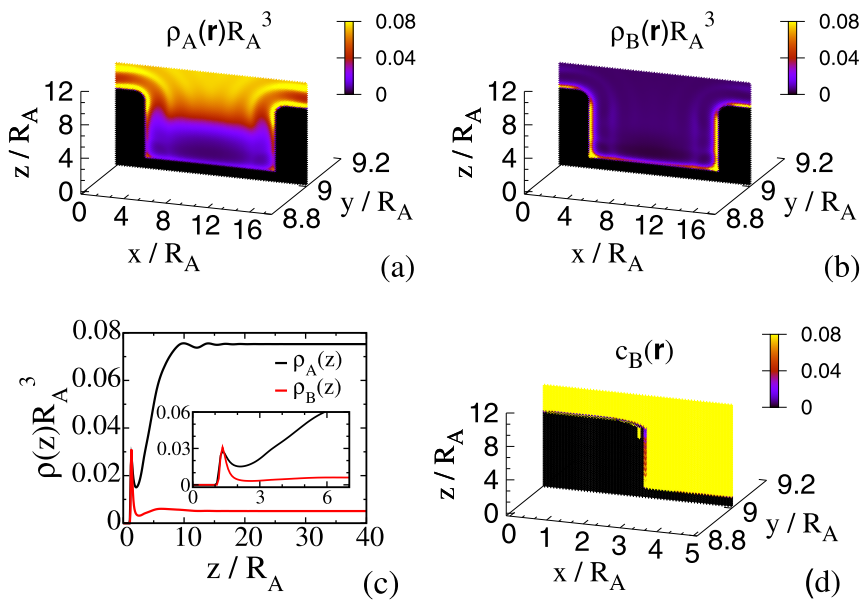

FIG. 15. Number densities $\rho_{A}(\mathbf{r})$ (a) and $\rho_{B}(\mathbf{r})$ (b) for the liquid with $c_{B} \approx 0.075$ and $\epsilon_{A B}=0.9 \times \epsilon_{A A}$, in the $x z$ plane passing through the center of the pit. Panel (c) shows the number density along a line parallel to the $z$ axis and passing through the center of the pit. For the given composition of the liquid phase the bulk liquid exhibits the packing fraction $\eta_{l}=0.3357660$, whereas the coexisting vapor has $\eta_{v}=0.0124869$. For this composition of the liquid bulk phase one has $\theta_{Y} \approx 86^{\circ}$. Panel (d) shows the concentration profile for the B particles in the same plane as shown in panels (a) and (b), focusing on those parts of the pit in which potentially interesting variations of $c_{B}$ occur. The fluid-wall interaction strengths and the pit dimensions are the ones used in Fig. 10. 
already intrudes at a contact angle substantially higher than $90^{\circ}$ for a ratio of the 0.84 of the A-B and A-A interaction strengths, whereas, if this ratio is chosen to be 1.0 , the contact angle has to be reduced considerably below $90^{\circ}$ until liquid intrudes the pits. For the shallow pits studied here, the contact angle, at which liquid intrudes, depends on the depth of the pit. This threshold contact angle $\theta_{i}$ for intrusion is smaller for deeper pits and in some cases even deviates more from the macroscopic prediction than for the shallow pits. These results demonstrate how the macroscopic capillary theory, which is based on interfacial free energies of extended interfaces, breaks down for nano-confinement. In the example discussed above, the threshold contact angle $\theta_{i}$ for intrusion of a binary liquid depends on the ratio $\epsilon_{A B} / \epsilon_{A A}$. For $\epsilon_{A B} / \epsilon_{A A}<1$ the tendency for demixing in the liquid phase at low temperatures increases as this ratio becomes smaller. (However, demixing under the conditions studied here is excluded.) It should be feasible to conduct experiments in which the composition of a binary liquid is varied along liquid-vapor coexistence until liquid intrudes into pits with suitably selected wall material. By selecting fluids with different "demixing tendencies" one could test the predicted variation of $\theta_{i}$. However, one should keep in mind that changing the fluid presumably changes the fluid-wall interactions, too, different from the clear-cut case studied in theory.

The free energy differences $\Delta \Omega$ between the Cassie and the Wenzel configuration, determined by using density functional theory (DFT), deviate quantitatively from the corresponding macroscopic predictions in terms of surface tensions. An improved agreement between the macroscopic predictions and the DFT results can be achieved if in the macroscopic expression for $\Delta \Omega$ a reduced effective width and a reduced effective depth of the pits is introduced, which are introduced by taking depletion zones into account. Nonetheless, substantial deviations persist. Surprisingly, nevertheless $\theta_{Y}^{c o}$ determined by using DFT is very close to the macroscopic prediction. All results of the above DFT analysis are not restricted to nanosized pits only, but also can be extended to binary colloidal suspensions intruding into micron-sized pits.

All computations presented here have been carried out at a fixed ratio $\epsilon_{A A} / k_{B} T=0.9834$, corresponding to temperatures well below the critical ones for all ratios $\epsilon_{A B} / \epsilon_{A A}$ studied. The influence of temperature on the Cassie-Wenzel transition is not studied explicitly, but only implicitly and, to a certain extent, via the dependence of the transition on Young's contact angle, which is temperature dependent. More detailed investigations of this issue are left to future studies. Furthermore, the present investigations have been limited to liquid-vapor coexistence. Pressures above the coexistence pressure tend to stabilize the Wenzel state. The analysis of pressure-induced Cassie-Wenzel transitions for binary liquid mixtures is also left to future studies.

At the end we would like to remark that thermally activated transitions between the Cassie and the Wenzel state could play a role for the narrow and shallow pits studied here. Although our results are based on density functional theory, which captures microscopic details but is of mean-field character, our results nonetheless provide the conditions in the vicinity of which thermally activated transitions may become noticeable.

\section{APPENDIX A: SPECIAL BINARY FLUID, CONTACT ANGLES, AND DENSITY PROFILES}

The special binary fluid which is a one-component liquid in the bulk $\left(R_{A}=R_{B}, \epsilon_{A A}=\epsilon_{A B}=\epsilon_{B B}\right)$, but a binary one with respect to the interaction with the wall, the macroscopic Young contact angle $\theta_{Y}$ on a corresponding planar wall computed as a function of composition is shown in Fig. 11. The interaction strength $\epsilon_{A}=0.9834 \times k_{B} T$ has been chosen such that the pure A liquid forms a contact angle $\theta_{Y}\left(c_{B}=0\right) \approx$ $112^{\circ}$. For the mixture with $c_{B} \neq 0$ a fixed ratio $\epsilon_{B} / \epsilon_{A}=2.333$ has been used. (In contrast to the contact angle measured from, e.g., a spherical-cap shaped sessile drop of finite size, the macroscopic Young contact angle $\theta_{Y}$ is independent of how the location of the interface is defined. For a detailed discussion of this issue see Ref. [51].) Increasing the concentration of B particles decreases the contact angle (see Fig. 11) as a result of the stronger interaction of the $\mathrm{B}$ particles with the wall.

Figure 12 shows the density distributions for a liquid composition of $c_{B} \approx 0.129$ corresponding to $\theta_{Y} \approx 83^{\circ}$. The configuration corresponds to the metastable Cassie state. If the concentration of $\mathrm{B}$ particles is increased further up to $c_{B} \approx 0.138$, which corresponds to $\theta_{Y} \approx 81^{\circ}$, the Cassie state becomes unstable. Both initial configurations (Cassie and Wenzel) eventually converge into the Wenzel state (Fig. 13). The number density of B particles is much higher at the walls compared with that of the A particles.

\section{APPENDIX B: BINARY FLUID, CONTACT ANGLE, AND NUMBER DENSITY PROFILES}

In Fig. 14, $\theta_{Y}$ is shown as a function of $\epsilon_{A B}$ for the bona fide binary liquid mixture at fixed composition $c_{B} \approx 0.05$. Upon

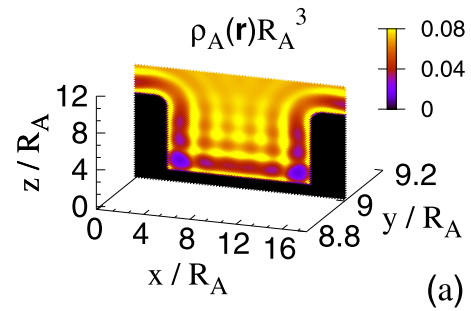

(a)
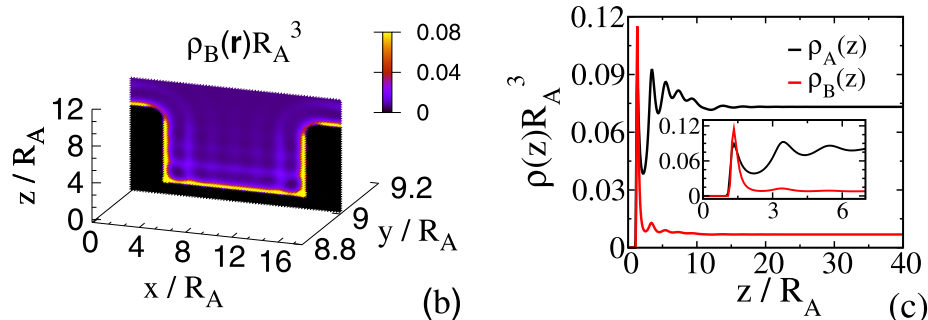

FIG. 16. The same as in Fig. 15 for $c_{B} \approx 0.085$ in the bulk liquid phase. Packing fractions of the coexisting liquid and vapor phases in the bulk are $\eta_{l}=0.3352940$ and $\eta_{v}=0.0128612$, respectively. The concentration of B particles in the bulk vapor phase is $c_{B} \approx 0.173$. The contact angle for the present system is $\theta_{Y} \approx 84^{\circ}$. 
lowering $\epsilon_{A B} / \epsilon_{A A}$, the contact angle decreases from $\theta_{Y} \approx 99^{\circ}$ at $\epsilon_{A B}=\epsilon_{A A}$ to $\theta_{Y} \approx 84^{\circ}$ for $\epsilon_{A B}=0.80 \times \epsilon_{A A}$.

For $\epsilon_{A B}=0.90 \times \epsilon_{A A}, w=7 \times \sigma$, and $D=4 \times \sigma$ the Cassie state remains metastable up to $c_{B} \approx 0.075$, for which the Wenzel state is the stable one. The corre- sponding Young's contact angle is $c a .86^{\circ}$. These values correspond to the number density profiles shown in Fig. 15. Upon increasing $c_{B}$ beyond 0.085 (Fig. 16), thereby decreasing $\theta_{Y}$ to $84^{\circ}$, the Cassie state becomes unstable.
[1] C. Neinhuis and W. Barthlott, Ann. Bot. 79, 667 (1997).

[2] X. Gao and L. Jiang, Nature (London) 432, 36 (2004).

[3] J. Genzer and A. Marmur, Mat. Res. Soc. Bull. 33, 742 (2008).

[4] Y. Wang and T. J. McCarthy, Langmuir 30, 2419 (2014).

[5] R. Blossey, Nat. Mater. 2, 301 (2003).

[6] E. Delamarche, D. Junker, and H. Schmid, Adv. Mater. 17, 2911 (2005).

[7] B. Bhushan, J. N. Israelachvilli, and U. Landman, Nature (London) 374, 607 (1995).

[8] D. Quéré, Annu. Rev. Mater. Sci. 38, 71 (2008).

[9] M. Heuberger, M. Zach, and N. D. Spencer, Science 292, 905 (2001).

[10] A. Giacomello, S. Meloni, M. Müller, and C. Casciola, J. Chem. Phys. 142, 104701 (2015).

[11] S. Khan and J. K. Singh, Mol. Simul. 40, 458 (2014).

[12] T. Yanagishita, K. Nishio, and H. Masuda, Adv. Mater. 17, 2241 (2005).

[13] E. Martines, K. Seunarine, H. Morgan, N. Gadegaard, C. D. W. Wilkinson, and M. O. Riehle, Nano Lett. 5, 2097 (2005).

[14] A. B. D. Cassie and S. Baxter, Trans. Faraday Soc. 40, 546 (1944).

[15] R. N. Wenzel, Ind. Eng. Chem. 28, 988 (1936).

[16] N. A. Ivanova, Z. B. Zhantenova, and V. M. Starov, Colloids Surf. A 413, 307 (2012).

[17] J. K. Borchardt, Colloids Surf. A 88, 13 (1994).

[18] T. F. Tadros, Applied Surfactants: Principles and Applications (Wiley, Weinheim, 2005).

[19] N. R. Biswal and S. Paria, Ind. Eng. Chem. Res. 50, 6138 (2011).

[20] X. Yao, Y. L. Song, and L. Jiang, Adv. Matter 23, 719 (2011).

[21] A. Tuteja, W. Choi, M. Ma, J. M. Mabry, S. A. Mazzella, G. C. Rutledge, G. H. McKinley, and R. E. Cohen, Science 318, 1618 (2007).

[22] W. Chen, A. Y. Fadeev, M. C. Hsieh, D. Öner, J. Youngblood, and T. J. McCarthy, Langmuir 15, 3395 (1999).

[23] C. Zhang, X. Zhao, J. Lei, Y. Ma, and F. Du, Soft Matter 13, 503 (2017).

[24] G. McDonnell and A. D. Russell, Clin. Microbiol. Rev. 12, 147 (1999).

[25] S. C. Laizure, T. Mandrell, N. M. Gades, and R. B. Parker, Drug Metab. Dispos. 31, 16 (2003).

[26] R. Hoogenboom, H. M. L. Thijs, D. Wouters, S. Hoeppener, and U. S. Schubert, Soft Matter 4, 103 (2008).

[27] H. Lin, H. Zhou, L. Xu, H. Zhu, and H. Huang, Biosyst. Eng. 143, 42 (2016).
[28] M. J. L. Castro, C. Ojeda, and A. F. Cirelli, Environ. Chem. Lett. 12, 85 (2014).

[29] R. J. L. Ramsey, G. R. Stephenson, and J. C. Haoo, Weed Sci. 54, 205 (2006).

[30] N. A. Ivanova and V. M. Starov, Curr. Opin. Colloid Interface Sci. 16, 285 (2011).

[31] A. Plech, U. Klemradt, M. Huber, and J. Peisl, Europhys. Lett. 49, 583 (2000)

[32] S. Dietrich and M. Schick, Phys. Rev. B 33, 4952 (1986).

[33] S. Dietrich, in Phase Transitions and Critical Phenomena, edited by C. Domb and J. L. Lebowitz (Academic, London, 1998), Vol. 12, p. 1.

[34] A. K. Mateya, S. Khan, and J. K. Singh, J. Phys. Chem. C 118, 4113 (2014).

[35] M. Lundgren, N. L. Allan, T. Cosgrove, and N. George, Langmuir 18, 10462 (2002).

[36] T. Koishi, K. Yasuoka, X. C. Zeng, and S. Fujikawad, Faraday Discuss. 146, 185 (2010).

[37] H. Tanaka, J. Phys.: Condens. Matter 13, 4637 (2001).

[38] B. Van Schaeybroeck and J. O. Indekeu, Phys. Rev. A 91, 013626 (2015).

[39] A. Giacomello, L. Schimmele, S. Dietrich, and M. Tasinkevych, Soft Matter 12, 8927 (2016).

[40] S. Meloni, A. Giacomello, and C. M. Casciola, J. Chem. Phys. 145, 211802 (2016).

[41] J. D. Weeks, D. Chandler, and H. C. Andersen, J. Chem. Phys. 54, 5237 (1971).

[42] S. L. Singh, L. Schimmele, and S. Dietrich, Phys. Rev. E 91, 032405 (2015).

[43] R. Evans, Adv. Phys. 28, 143 (1979).

[44] Y. Singh, Phys. Rep. 207, 351 (1991).

[45] R. Roth, R. Evans, A. Lang, and G. Kahl, J. Phys.: Condens. Matter 14, 12063 (2002).

[46] P. Tarazona, J. A. Cuesta, and Y. Martìnez-Ratòn, in Theory and Simulation of Hard-Sphere Fluids and Related Systems, edited by Á. Mulero, Lecture Notes in Physics Vol. 753 (Springer, Heidelberg, 2008), p. 247.

[47] Y. Rosenfeld, Phys. Rev. Lett. 63, 980 (1989).

[48] R. Roth, J. Phys.: Condens. Matter 22, 063102 (2010).

[49] Y. Rosenfeld, M. Schmidt, H. Löwen, and P. Tarazona, Phys. Rev. E 55, 4245 (1997).

[50] H. Hansen-Goos and R. Roth, J. Phys.: Condens. Matter 18, 8413 (2006).

[51] L. Schimmele, M. Napiórkowski, and S. Dietrich, J. Chem. Phys. 127, 164715 (2007). 